\title{
Metamodel-based multidisciplinary design optimization methods for aerospace system
}

\author{
Renhe Shi ${ }^{1,2}$, Teng $\operatorname{Long}^{1,2}(\bowtie)$, Nianhui Ye ${ }^{1,2}$, Yufei Wu ${ }^{1,2}$, Zhao Wei ${ }^{1,2}$, and Zhenyu Liu ${ }^{1,2}$ \\ 1. School of Aerospace Engineering, Beijing Institute of Technology, Beijing 100081, China \\ 2. Key Laboratory of Dynamics and Control of Flight Vehicle, Ministry of Education, Beijing 100081, China
}

\section{ABSTRACT}

The design of complex aerospace systems is a multidisciplinary design optimization (MDO) problem involving the interaction of multiple disciplines. However, because of the necessity of evaluating expensive black-box simulations, the enormous computational cost of solving MDO problems in aerospace systems has also become a problem in practice. To resolve this, metamodel-based design optimization techniques have been applied to MDO. With these methods, system models can be rapidly predicted using approximate metamodels to improve the optimization efficiency. This paper presents an overall survey of metamodelbased MDO for aerospace systems. From the perspective of aerospace system design, this paper introduces the fundamental methodology and technology of metamodelbased MDO, including aerospace system MDO problem formulation, metamodeling techniques, state-of-the-art metamodel-based multidisciplinary optimization strategies, and expensive black-box constraint-handling mechanisms. Moreover, various aerospace system examples are presented to illustrate the application of metamodel-based MDOs to practical engineering. The conclusions derived from this work are summarized in the final section of the paper. The survey results are expected to serve as guide and reference for designers involved in metamodel-based MDO in the field of aerospace engineering.

\section{KEYWORDS}

aerospace systems design

multidisciplinary design

optimization (MDO)

metamodel-based design and optimization (MBDO)

expensive black-box problems

\section{Review Article}

Received: 14 July 2021

Accepted: 23 July 2021

(C) The Author(s) 2021

\section{Introduction}

In practice, the development of aerospace systems involves sophisticated system engineering. Aerospace systems (e.g., satellites and spacecraft) are multidisciplinary schemes consisting of several inter-coupled disciplines or subsystems, including orbit, power, propulsion, structure, thermal control, attitude control, and payload. Multidisciplinary design optimization (MDO) has been widely employed in engineering systems design practices to improve design performance and reduce design costs in the early development process [1]. The concept of MDO was initially developed by Sobieszczanski-Sobieski [2]. As a methodology, MDO focuses on the design of complex engineering systems and subsystems by coherently exploiting the synergy of mutually interacting phenomena [2]. It comprehensively utilizes various computational analysis tools and optimization methods to determine the optimal

凶 tenglong@bit.edu.cn design of systems [3]. Moreover, MDO was first applied to the coupled aero-structural optimization problem for aircraft wings [4]. The merits of MDO have been widely proven in the design practices of engineering systems, such as aircraft [5-10], automobiles [11-13], ships [14,15], and electric devices $[16,17]$. In the past decade, MDO has also been applied to aerospace systems, including satellites [18-21], constellations [20,22,23], and launch rockets [24-27], to improve the overall system performance.

Although deriving the optimal design of multidisciplinary systems is advantageous, the implementation of MDO is confronted with two crucial problems in practical engineering. The basic problem involves the means by which multiple disciplines are to be coupled. The simplest approach is to implement multidisciplinary analysis (MDA) during optimization, where the coupling

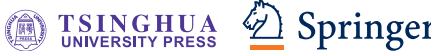


variables are directly solved via iterative analysis. In addition, several MDO architectures have been successfully developed to organize different analysis models and computational flow for multidisciplinary optimization. These architectures can be generally classified as monolithic (e.g., all-at-once, individual discipline feasible, and multidisciplinary feasible) architectures and distributed (e.g., cooperative optimization and concurrent subspace optimization) architectures [3], with respect to whether sub-optimization is required. Another critical problem involves achieving efficiency in solving MDO problems with limited computational resource. Modern aerospace system designs typically employ high-fidelity disciplinary simulation models (e.g., finite element analysis (FEA) model with large grids) to enhance the quality and reliability of design optimization results. However, the evaluation of expensive simulation models and iterative MDA computing processes further considerably increase the associated computational cost of MDO problems in aerospace systems. Moreover, because of the lack of transparency in simulation models (e.g., simulations implemented using commercial computer-aided engineering (CAE) software), the MDA process is generally referred to as an expensive black-box function, whose gradient is expensive or unreliable for calculation. Thus, most existing heuristic or gradient-based numerical methods (e.g., genetic algorithm and sequential quadratic programming) are unsuitable for solving computation-intensive blackbox optimization problems.

To reduce the computational cost of expensive blackbox optimization problems, metamodel-based design optimization (MBDO) techniques have been developed over the past two decades [28]. These methods are also called surrogate-assisted analysis and optimization methods [29]. They involve the construction of a metamodel (or a surrogate) based on a set of samples to approximate the original expensive simulation models for analysis or optimization. These MBDO methods can also be applied to solve MDO problems. In this case, an MDO problem is essentially treated as a general constrained nonlinear optimization problem, where the computationally intensive MDA process is replaced with metamodels to reduce the number of expensive function calls. In recent years, several state-of-the-art MBDO methods have been developed [30]. Different MBDO methods are distinguished by their metamodeling techniques and design space exploration strategies. The combination of the MBDO and MDO methodologies can be referred to as metamodel-based MDO. Note that in practice, the purpose of metamodel-based MDO is to overcome the burden of optimization rather than build or solve MDO architectures.

A number of reviews regarding $\mathrm{MDO}$ have been reported in the literature $[1,3,31,32]$. Many surveys on metamodel-based design and optimization have also been conducted in recent years [28, 29,33]. However, comprehensive surveys on metamodel-based MDOs are rarely performed $[34,35]$. Focusing on the design of aerospace systems, this paper introduces the fundamental methodology and technology of state-of-the-art metamodel-based MDO techniques. The remainder of this paper is organized as follows. Section 2 introduces the problem formulation and overall architecture of the metamodelbased MDO. The common metamodeling techniques are described in Section 3. In Section 4, the metamodelbased multidisciplinary optimization strategies are discussed in detail. The survey of expensive black-box constraint-handling mechanisms for metamodel-based MDOs is presented in Section 5. Moreover, several realworld aerospace system MDO examples are discussed in Section 6. Finally, some conclusions and future works on metamodel-based MDOs are summarized in Section 7.

\section{Methodology of metamodel-based MDO}

\subsection{Formulation of aerospace system MDO problem}

The development of an aerospace system is a complex system engineering task. For instance, a satellite system consists of multiple inter-coupled disciplines, including orbit, propulsion, attitude control, payload, and structure, as shown in Fig. 1. To improve system performance,

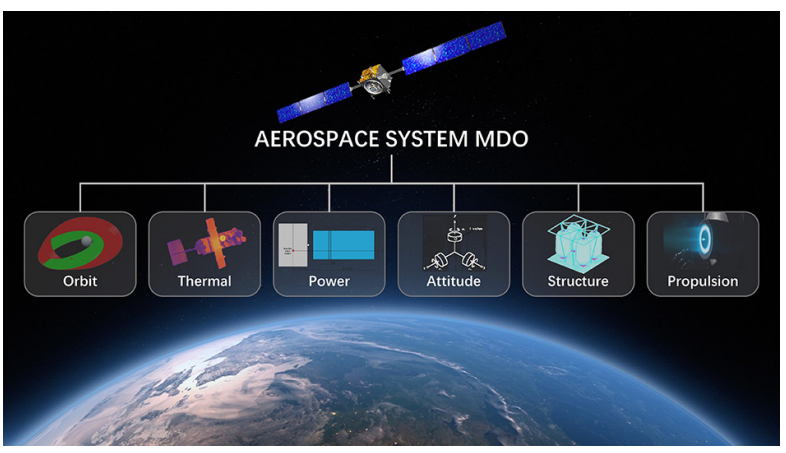

Fig. 1 Various disciplines for aerospace systems. 
MDO was employed to determine the optimal design parameters for the aerospace system.

In MDO, the inter-coupled relationships, data exchange, and simulation flow among different disciplines can be organized in terms of the design structure matrix, as illustrated in Fig. 2. In the matrix, the diagonal elements (i.e., shaded blocks) represent different disciplines involved in the aerospace system. The terms above the diagonal represent feed-forward variables and parameters, whereas those below represent backward information. The design structure matrix explicitly expresses the constitution of the MDO problem. Based on the constructed matrix, the general mathematical formulation of an aerospace system MDO problem can be represented as

$$
\begin{array}{ll}
\min & f(\boldsymbol{X}), \boldsymbol{X}=\left[\boldsymbol{X}_{1}, \boldsymbol{X}_{2}, \cdots, \boldsymbol{X}_{N_{\mathrm{d}}}\right]^{\mathrm{T}} \\
\text { s.t. } & \begin{cases}D_{i}\left(\boldsymbol{X}_{i}, \boldsymbol{Y}_{i}, \boldsymbol{Y}_{i j}\right)=0, \quad i=1,2, \cdots, N_{\mathrm{d}} \\
g_{i}\left(\boldsymbol{X}_{i}, \boldsymbol{Y}_{i}, \boldsymbol{Y}_{i j}\right) \leqslant 0, \quad i=1,2, \cdots, N_{\mathrm{d}} \\
\boldsymbol{X}_{\mathrm{LB}} \leqslant \boldsymbol{X} \leqslant \boldsymbol{X}_{\mathrm{UB}}\end{cases}
\end{array}
$$

where $f(\boldsymbol{X})$ is the objective of the aerospace system MDO problem (e.g., total mass and lifecycle cost); $N_{\mathrm{d}}$ is the number of disciplines; $\boldsymbol{X}$ is the vector of design variables; $\boldsymbol{X}_{i}$ and $\boldsymbol{Y}_{i}$ are the design variables and output (i.e., state variables) of the $i$ th discipline, respectively; $\boldsymbol{X}_{\mathrm{LB}}$ and $\boldsymbol{X}_{\mathrm{UB}}$ are the lower and upper bounds of design variable, respectively; $\boldsymbol{Y}_{i j}$ is the coupling state variables from the $i$ th discipline to the $j$ th discipline; and $D_{i}\left(\boldsymbol{X}_{i}, \boldsymbol{Y}_{i}, \boldsymbol{Y}_{i j}\right)$ and $g_{i}\left(\boldsymbol{X}_{i}, \boldsymbol{Y}_{i}, \boldsymbol{Y}_{i j}\right)$ are the state equations (i.e., disciplinary analysis model) and constraints of the $i$ th discipline, respectively.

Note that because of feedback coupling state variables, $\boldsymbol{Y}_{\mathrm{FV}}=\left\{\boldsymbol{Y}_{i j} \mid i>j\right\}$, the MDA process must derive a consistent solution at each sample point during the metamodel-based optimization process. This means that the solution $\left(D_{i}\left(\boldsymbol{X}_{i}, \boldsymbol{Y}_{i}, \boldsymbol{Y}_{i j}\right), i=1,2, \cdots, N_{\mathrm{d}}\right)$ must satisfy all state equations. In practice, the MDA process can be organized via a fixed-point-based iteration approach, as summarized in Algorithm 1.

\subsection{Overall procedure of metamodel-based optimization}

Because modern aerospace system design generally involves computationally expensive simulation models, MBDO techniques are employed to solve MDO problems to reduce the computational cost. In the metamodelbased MDO process, the entire MDA process is evaluated

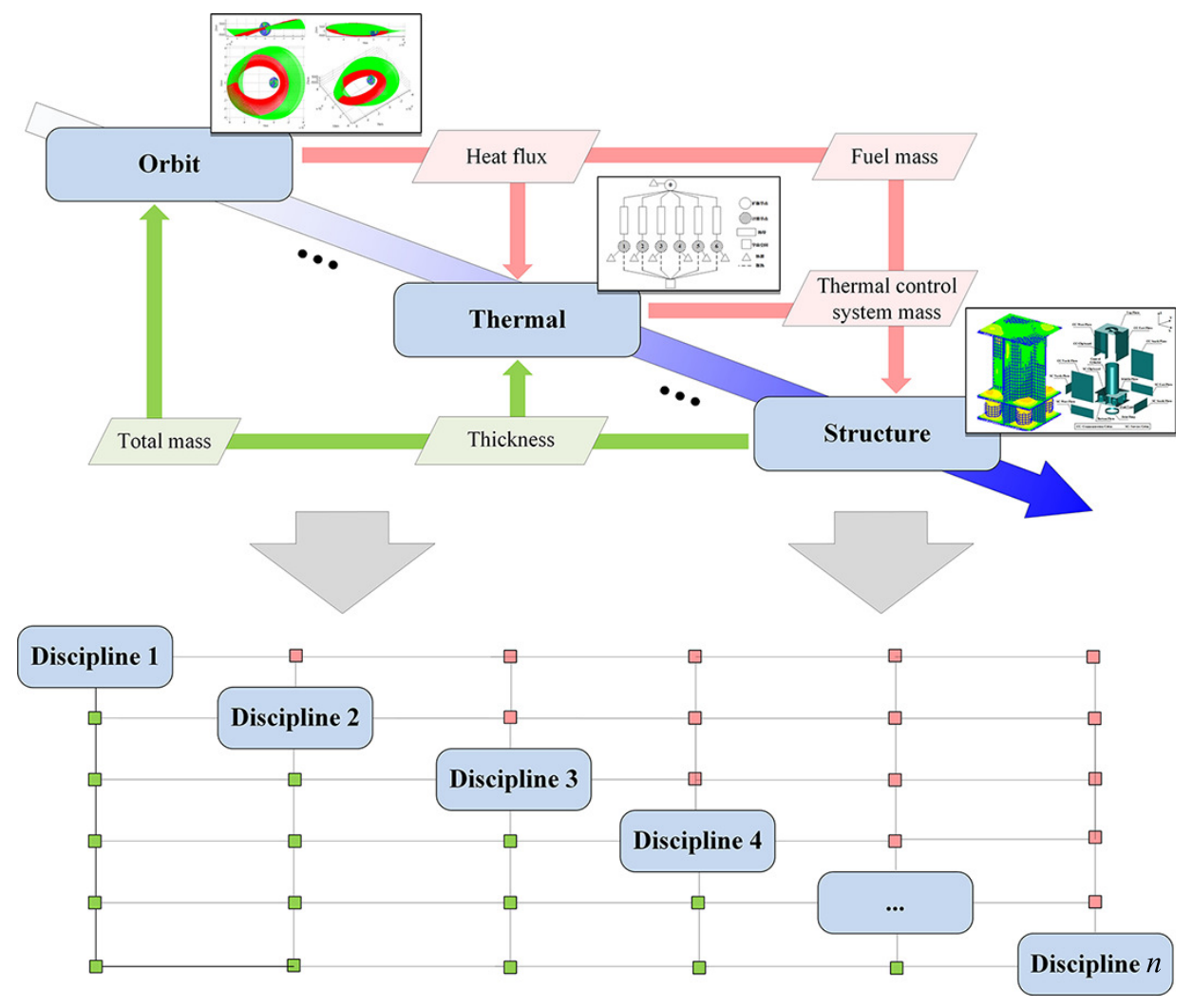

Fig. 2 Design structure matrix of aerospace system MDO problem. 


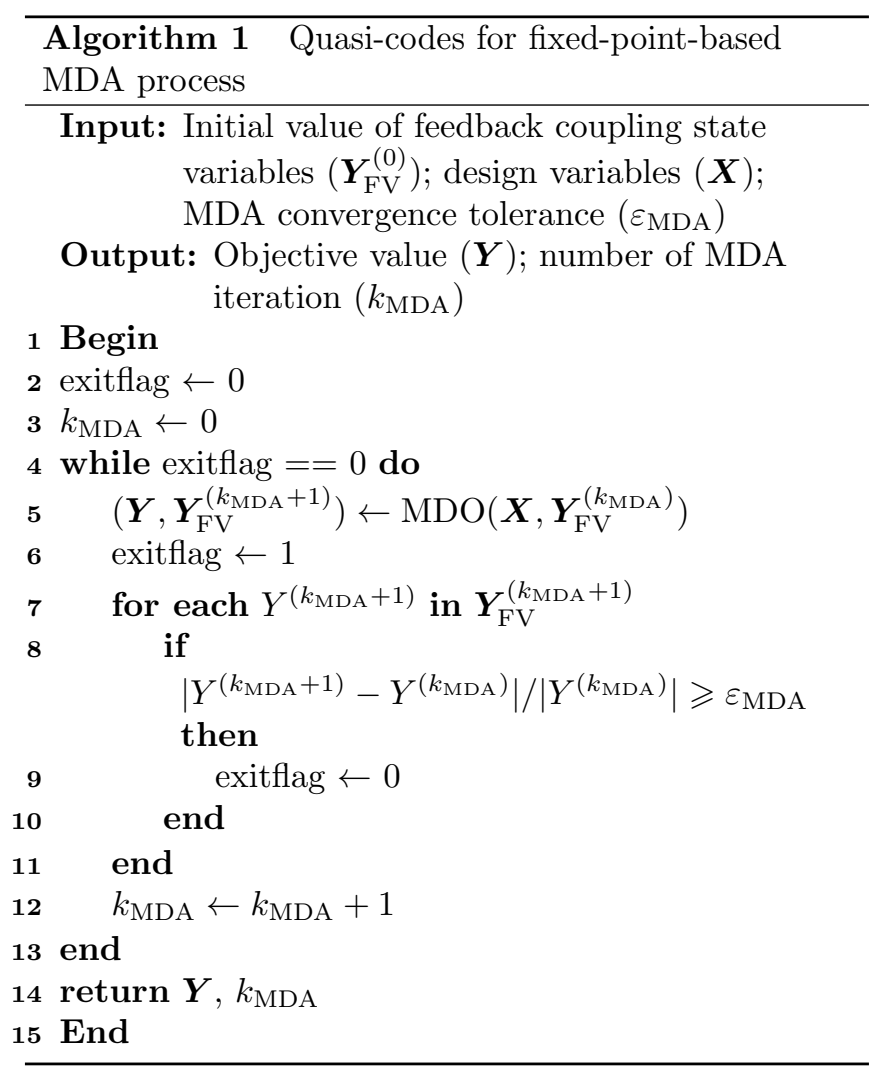

to obtain a consistent design at each sample point. The metamodels are constructed to approximate the MDA process for multidisciplinary optimization. In this case, the responses of expensive black-box objectives and constraints can be rapidly and inexpensively predicted by metamodels. Note that metamodels can be gradually refined via adaptive sampling during the optimization process to further reduce the computational cost. Moreover, Fig. 3 illustrates the overall procedure of metamodelbased aerospace system MDO [19]. The major steps are as follows:

Step 1: The aerospace system MDO problem, including the design space, objective, constraints, and MDA models to be optimized, is clearly defined. The algorithm parameters (e.g., the number of initial samples and termination criterion) of the selected MBDO methods for solving the MDO problem are configured.

Step 2: A number of sample points are generated by the design of the experiment in the design space. Then, the MDA process is invoked to obtain the objective and constraint responses at each sample point. The sample points and their associated responses (referred to

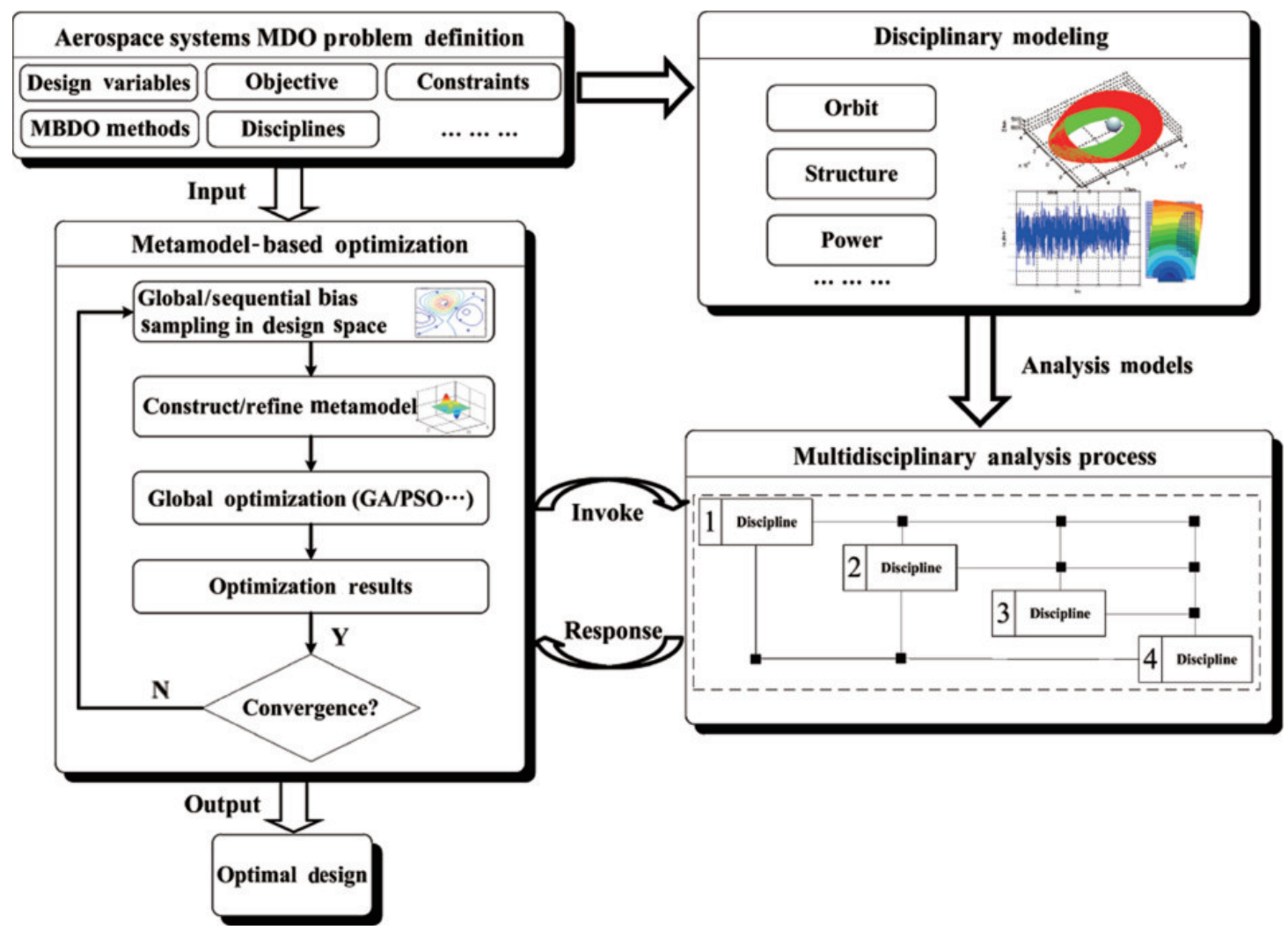

Fig. 3 Overall procedure of metamodel-based MDO for aerospace systems (reproduced with permission from Ref. [19], C) IAA 2017).

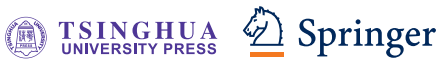


as samples) are stored in the sample dataset.

Step 3: Based on existing samples in the sample dataset, metamodels are constructed to approximate the objective and constraint responses from the costly MDA process. If the static metamodel-based optimization strategy is used, then the approximation accuracy of metamodels must be verified, as discussed in Section 2.3.

Step 4: The constructed metamodels are used to replace the original expensive MDA process for optimization. Global numerical optimization techniques, such as genetic algorithms, are employed to directly optimize the metamodels instead of the original expensive MDA models, as given by Eq. (2):

$$
\begin{array}{ll}
\min & \hat{f}(\boldsymbol{X}), \boldsymbol{X}=\left[\boldsymbol{X}_{1}, \boldsymbol{X}_{2}, \cdots, \boldsymbol{X}_{\mathrm{n}}\right]^{\mathrm{T}} \\
\text { s.t. } & \left\{\begin{array}{l}
\hat{g}_{i}(\boldsymbol{X}) \leqslant 0, \\
\boldsymbol{X}_{\mathrm{LB}} \leqslant \boldsymbol{X} \leqslant \boldsymbol{X}_{\mathrm{UB}}
\end{array}\right.
\end{array}
$$

where $m$ is the number of constraints, and $\hat{f}(\boldsymbol{X})$ and $\hat{g}_{i}(\boldsymbol{X})$ are the metamodels of the objective and the $i$ th constraint, respectively. If the dynamic metamodel-based optimization strategy is used, the metamodels are adaptively refined during optimization to efficiently search for the optimum, as discussed in detail in Section 4.

Step 5: The termination criterion is checked to determine whether the optimization must be terminated. The common termination criteria in metamodel-based MDO include the decrement criterion $\left(C_{1}\right)$ and computational cost criterion $\left(C_{2}\right)$ [36], as given in Eq. (3). In $C_{1}$, if the error or relative error between the objective values in two consecutive iterations is less than the tolerance $\left(\varepsilon_{\mathrm{OPT}}\right)$, then the optimization terminates. In $C_{2}$, if the number of existing samples $\left(N_{\mathrm{e}}\right)$ exceeds the predefined maximum number of function evaluations $\left(\mathrm{NFE}_{\max }\right)$, then the optimization terminates.

$$
\begin{aligned}
& C_{1}:\left(\left|\frac{f\left(\boldsymbol{X}^{(k)}\right)-f\left(\boldsymbol{X}^{(k-1)}\right)}{f\left(\boldsymbol{X}^{(k)}\right)}\right| \leqslant \varepsilon_{\mathrm{OPT}} \|\right. \\
& \left.\quad\left|f\left(\boldsymbol{X}^{(k)}\right)-f\left(\boldsymbol{X}^{(k-1)}\right)\right| \leqslant \varepsilon_{\mathrm{OPT}}\right) \\
& C_{2}: N_{\mathrm{e}}>\mathrm{NFE}_{\max }
\end{aligned}
$$

Step 6: The optimized design for the aerospace system MDO problem is finally obtained when the termination criterion is reached. For the single-objective optimization problem, the best feasible solution in the sample dataset is outputted as the final solution. For the multi-objective optimization problem, the non-dominated designs in the sample dataset are outputted as a Pareto solution.

\subsection{Architecture of metamodel-based MDO techniques}

\subsubsection{Classification of metamodel-based MDO methods}

Metamodel-based MDO methods may be generally classified into two categories in terms of whether the metamodels are updated during optimization: static and dynamic metamodel-based optimization strategies (Fig. 4) [36].

In the static metamodel-based optimization strategy, metamodels are constructed once based on sufficient samples for optimization. This is the most convenient approach to implement the metamodel-based MDO process. For instance, some expensive disciplinary models can be replaced with well-constructed metamodels for MDA and optimization. Note that to ensure the confidence in their results, the approximation accuracy of the constructed metamodels should be verified before optimization. Techniques that are widely used for accuracy validation include split and crossover validation methods. In the split validation method, the samples are divided into two groups. One group of samples is applied to construct the metamodels, and the other is used to validate their accuracy. For the crossover validation method, several samples are randomly selected for validation, whereas the remaining samples are used for metamodeling. The validation process is repeated until the prediction errors in all the samples are obtained. The most commonly used approximation accuracy indices for metamodels are summarized in Table 1 [36]. For example, if the complex correlation coefficient $\left(R^{2}\right)$ exceeds 0.9 , then the metamodel can be assumed to be sufficiently accurate for optimization.

In contrast to constructing a static metamodel once, the metamodels in the dynamic metamodel-based optimization strategy are adaptively refined according to certain criteria or mechanisms during optimization. Overall, the dynamic metamodel-based optimization strategy can be further divided into infill sampling criterion-based methods and space reduction-based methods. Compared with the static metamodel-based optimization strategy, the dynamic metamodel-based optimization strategy, which has become popular in recent years, is generally more efficient in practice. This study mainly focuses on surveying the dynamic metamodel-based optimization strategy, as discussed in detail in Section 4.

(19.9.9. 


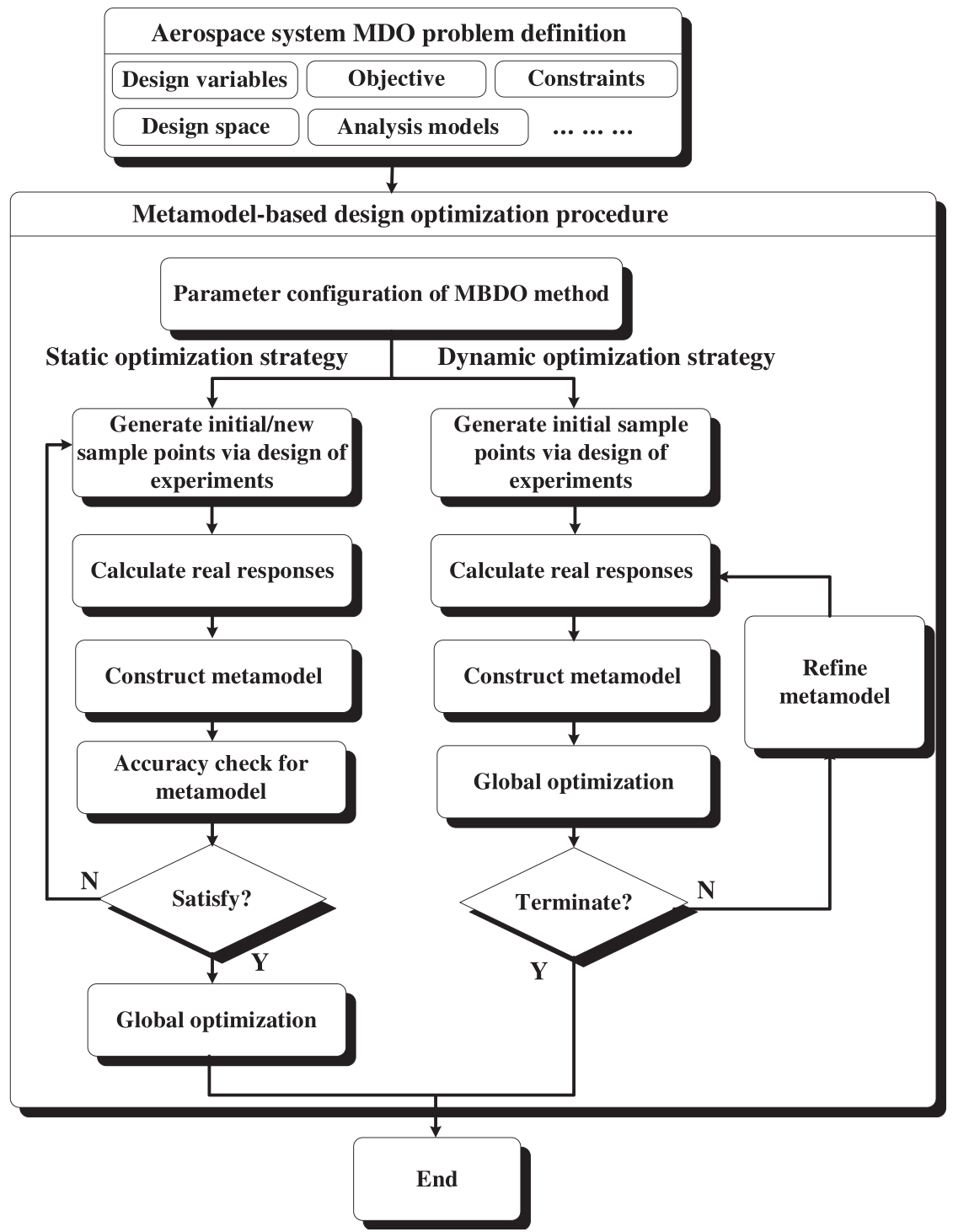

Fig. 4 Classification of MBDO methods.

Table 1 Approximation accuracy indices for metamodel validation

\begin{tabular}{|c|c|c|}
\hline Index & Symbol & Formulation \\
\hline Complex correlation coefficient & $R^{2}$ & $R^{2}=1-\sum_{i=1}^{N_{\text {test }}}\left(y_{i}^{(\text {test })}-\hat{y}_{i}^{(\text {test })}\right)^{2} / \sum_{i=1}^{N_{\text {test }}}\left(y_{i}^{(\text {test })}-\bar{y}^{(\text {test })}\right)^{2}$ \\
\hline Root mean square error & RMSE & $\mathrm{RMSE}=\sqrt{\frac{1}{N_{\text {test }}} \sum_{i=1}^{N_{\text {test }}}\left(y_{i}^{(\text {test })}-\hat{y}_{i}^{(\text {test })}\right)^{2}}$ \\
\hline Relative average absolute error & RAAE & 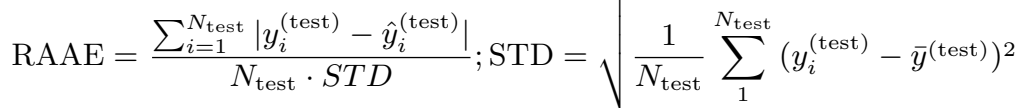 \\
\hline Relative maximum absolute error & RMAE & $\operatorname{RMAE}=\frac{\max \left(\left|y_{1}^{(\text {test })}-\bar{y}^{\text {(test) }}\right|,\left|y_{2}^{(\text {test })}-\bar{y}^{(\text {test })}\right|, \cdots,\left|y_{N_{\text {test }}}^{\text {(test })}-\bar{y}^{\text {(test })}\right|\right)}{\operatorname{STD}}$ \\
\hline
\end{tabular}

Note: $N_{\text {test }}$ is the number of test samples; $y_{i}^{\text {(test) }}$ is the real response of the $i$ th test sample; $\hat{y}_{i}^{(\text {test })}$ is the predicted response of the $i$ th test sample; $\bar{y}^{\text {(test) }}$ is the mean response of the test samples. 


\subsubsection{Diagram of metamodel-based MDO archi- tecture}

Based on the foregoing discussion, Fig. 5 illustrates the architecture of the metamodel-based MDO technique [36]. Before optimization, the MDA models of aerospace systems (e.g., orbit, structure, and attitude control) must be established and parameterized. Then, MBDO techniques are employed to optimize the MDO problem by evaluating the MDA process. During the optimization, metamodels are constructed and refined to explore the design space. The optimization stops when the termination criterion is satisfied. The optimized solution is outputted for further analysis.

\section{Metamodeling techniques}

\subsection{Design of experiments}

The design of experiments aims at generating sample points in the design space to represent the numerical characteristics of the system. Considering the limited computational resource in practice, the design of experimental methods is expected to provide favorable projective uniformity and space-filling uniformity performance. In the metamodel-based aerospace system MDO process, Latin hypercube is the most commonly used design among experimental methods. It can generate sample sets with arbitrary quantities and dimensions. To further improve the sampling performance, in recent years, optimal Latin hypercube design methods have been developed based on certain criteria, such as minimum distance [37], entropy [38], and energy [39]. For instance, two typical optimal Latin hypercube design methods, that is, the native lhsdesign function in the MATLAB optimization toolbox [40] and ESEA-OLHD [41], are illustrated in Fig. 6. The lhsdesign function is implemented by randomly generating several groups of sample points and selecting the group with the best space-filling ability. Moreover, ESEA-OLHD generates sample points by optimizing the space-filling criteria via numerical optimization algorithms. This shows that the random sample points generated by the optimal Latin hypercube design methods can uniformly fill the design space, thus improving the metamodeling performance in practice. In recent years, some novel OLHD methods specific for metamodelbased optimization have been developed [42-44].

After the generation of sample points, the associated responses at each sample point are evaluated by calling the MDA process. The samples are then utilized to construct metamodels for MDO.

\subsection{Typical metamodels}

\subsubsection{Polynomial response surface method}

The polynomial response surface method constructs a multivariate linear regression function to fit the costly simulation model or MDA process [45]. A polynomial response surface metamodel can be written as

$$
\begin{aligned}
\hat{f}_{\mathrm{PRSM}}(\boldsymbol{x})= & \beta^{(0)}+\sum_{i=1}^{N_{\mathrm{v}}} \beta^{(i)} x^{(i)} \\
& +\sum_{i=1}^{N_{\mathrm{v}}} \beta^{(i i)}\left(x^{(i)}\right)^{2}+\sum_{i=1}^{N_{\mathrm{v}}} \sum_{j>1}^{N_{\mathrm{v}}} \beta^{(i j)} x^{(i)} x^{(j)}
\end{aligned}
$$

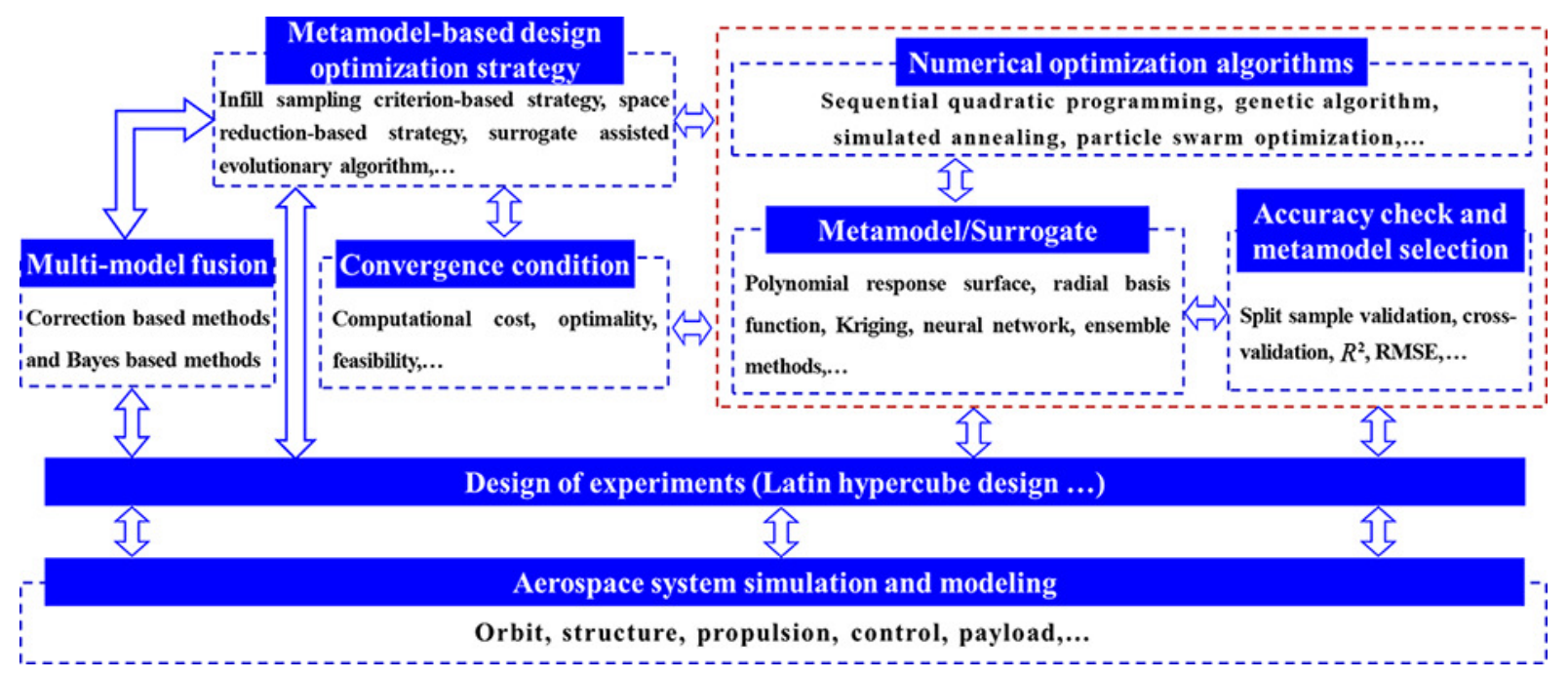

Fig. 5 Diagram of metamodel-based MDO architecture. 


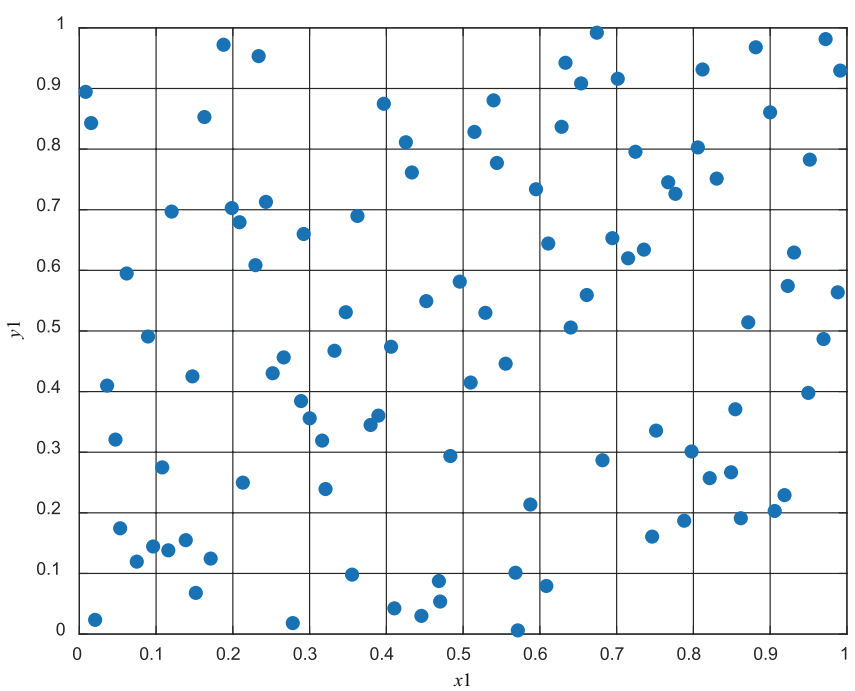

(a) lhsdesign

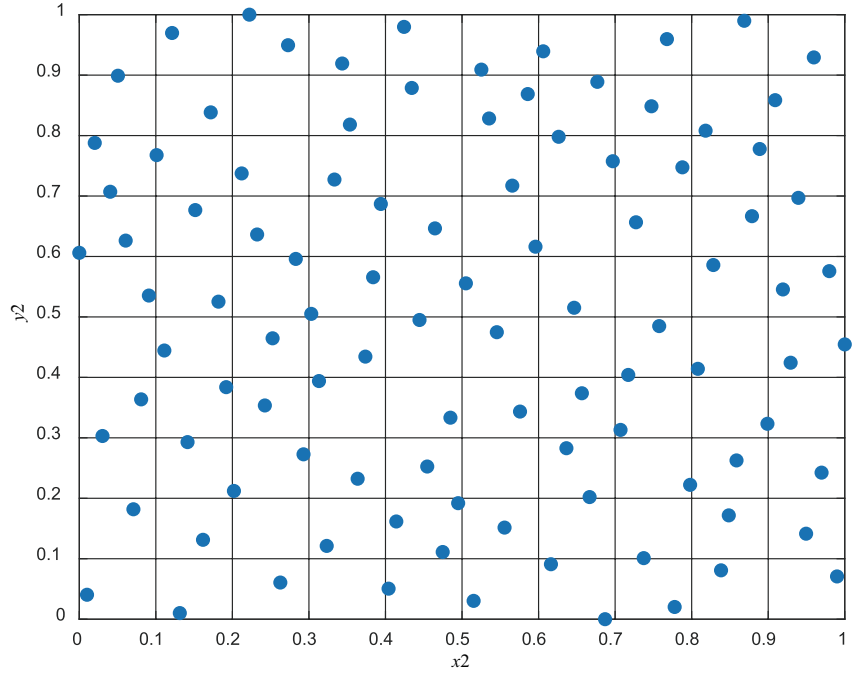

(b) ESEA-OLHD

Fig. 6 Various optimal Latin hypercube design methods.

where $N_{\mathrm{v}}$ is the dimensionality of design variables; $\beta^{(0)}, \beta^{(i)}, \beta^{(i j)}$ are the coefficients estimated through the least squares method. The coefficient matrix, $\boldsymbol{\beta}$, is given by

$$
\boldsymbol{\beta}=\left(\boldsymbol{\Phi}^{\mathrm{T}} \boldsymbol{\Phi}\right)^{-1} \boldsymbol{\Phi}^{\mathrm{T}} \boldsymbol{y}
$$

where $\boldsymbol{y}$ is the response vector of training sample points, and $\boldsymbol{\Phi}$ is the matrix relevant to the training sample points.

\subsubsection{Radial basis functions}

Radial basis function is an interpolation method based on the function value at sample points [46]. A radial basis function metamodel can be formulated as

$$
\hat{f}_{\mathrm{RBF}}(\boldsymbol{x})=\sum_{i=1}^{N_{\mathrm{t}}} \omega_{i} \phi_{\mathrm{r}}\left(\left\|\boldsymbol{x}-\boldsymbol{x}_{i}\right\|\right)
$$

where $N_{\mathrm{t}}$ is the number of training sample points; $\phi_{\mathrm{r}}\left(\left\|\boldsymbol{x}-\boldsymbol{x}_{i}\right\|\right), i=1,2, \cdots, N_{\mathrm{t}}$, is the radial function; and $\omega_{i}$ is the weight coefficient of radial function. The coefficient vector $(\boldsymbol{\omega})$ can be calculated as follows:

$$
\begin{aligned}
\boldsymbol{\omega} & =\boldsymbol{A}^{-1} \boldsymbol{y} \\
\boldsymbol{y} & =\left[y_{1}, y_{2}, \cdots, y_{N_{\mathrm{t}}}\right]^{\mathrm{T}} \\
\boldsymbol{A} & =\left[\begin{array}{ccc}
\phi\left(\left\|\boldsymbol{x}_{1}-\boldsymbol{x}_{1}\right\|\right) & \cdots & \phi\left(\left\|\boldsymbol{x}_{1}-\boldsymbol{x}_{N_{\mathrm{t}}}\right\|\right) \\
\vdots & \ddots & \vdots \\
\phi\left(\left\|\boldsymbol{x}_{N_{\mathrm{t}}}-\boldsymbol{x}_{1}\right\|\right) & \cdots & \phi\left(\left\|\boldsymbol{x}_{N_{\mathrm{t}}}-\boldsymbol{x}_{N_{\mathrm{t}}}\right\|\right)
\end{array}\right]_{N_{\mathrm{t}} \times N_{\mathrm{t}}}
\end{aligned}
$$

The typically used radial functions are listed in Table 2 [36], where is the Euclidean distance is $\left\|\boldsymbol{x}-\boldsymbol{x}_{i}\right\|$.

The approximate accuracy of this metamodel is influenced by shape coefficient $c$, which is determined by the
Table 2 Commonly used radial functions

\begin{tabular}{cc}
\hline Function & Formulation \\
\hline Linear & $\phi(r)=(r+c)$ \\
Gauss & $\phi(r)=\exp \left(-c r^{2}\right)$ \\
Spline & $\phi(r)=r^{2} \log \left(c r^{2}\right)$ \\
Cubic & $\phi(r)=(r+c)^{3}$ \\
Multiquadratic & $\phi(r)=\left(r^{2}+c^{2}\right)^{1 / 2}$ \\
Inverse multiquadratic & $\phi(r)=\left(r^{2}+c^{2}\right)^{-1 / 2}$ \\
\hline
\end{tabular}

distribution of sample points and function information. Typically, $c$ can be estimated using Eq. (8) [36]:

$$
c=\left((\max (\boldsymbol{x})-\min (\boldsymbol{x})) / N_{\mathrm{t}}\right)^{\frac{1}{N_{\mathrm{v}}}}
$$

\subsubsection{Kriging}

The Kriging model is a type of unbiased optimal estimation interpolation model that combines a global approximation model and a stochastic process. It is formulated using Eq. (9):

$$
\hat{f}_{\mathrm{KRG}}(\boldsymbol{x})=\mu(\boldsymbol{x})+Z(\boldsymbol{x})
$$

where $\mu(\boldsymbol{x})$ is the global approximation model, which reflects the variation trend of the expensive MDA process and is usually set as a constant; $Z(\boldsymbol{x})$ represents a Gaussian process with zero mean and variance $\left(\sigma^{2}\right)$. Given a set of sample points, $\boldsymbol{X}=\left\{\boldsymbol{x}_{1}, \boldsymbol{x}_{2}, \cdots, \boldsymbol{x}_{N_{\mathrm{t}}}\right\}$, the covariance matrix is given by Eq. (10):

$$
\operatorname{Cov}(Z(\boldsymbol{X}))=\sigma^{2} \boldsymbol{R}\left(R\left(\boldsymbol{x}_{i}, \boldsymbol{x}_{j}\right)\right), i, j=1,2, \cdots, N_{\mathrm{t}}
$$

where $\boldsymbol{R}(\cdot)$ is a symmetric correlation matrix, and $R\left(\boldsymbol{x}_{i} \boldsymbol{x}_{j}\right)$ is the Gaussian correlation function between 
sample points $\boldsymbol{x}_{i}$ and $\boldsymbol{x}_{j}$, as shown in Eq. (11):

$$
R\left(\boldsymbol{x}_{i}, \boldsymbol{x}_{j}\right)=\exp \left(-\sum_{k=1}^{N_{\mathrm{v}}} \theta_{k}\left|x_{i}^{(k)}-x_{j}^{(k)}\right|^{2}\right)
$$

where $\theta_{k}$ is the correlation parameter determined by maximizing the likelihood function in Eq. (12):

$$
L(\boldsymbol{x})=-\frac{N_{\mathrm{t}}}{2} \ln \left(\hat{\sigma}^{2}\right)-\frac{1}{2} \ln (|R|)
$$

The estimated variance $\left(\hat{\sigma}^{2}\right)$ and mean values $(\hat{\mu})$ can be calculated using Eq. (13):

$$
\begin{aligned}
\hat{\mu} & =\frac{\mathbf{1}^{\mathrm{T}} \boldsymbol{R}^{-1} \boldsymbol{Y}_{T}}{\mathbf{1}^{\mathrm{T}} \boldsymbol{R}^{-1} \mathbf{1}} \\
\hat{\sigma}^{2} & =\frac{\left(\boldsymbol{Y}_{T}-\mathbf{1} \hat{\mu}\right)^{\mathrm{T}} \boldsymbol{R}^{-1}\left(\boldsymbol{Y}_{T}-\mathbf{1} \hat{\mu}\right)}{N_{\mathrm{t}}}
\end{aligned}
$$

where $\boldsymbol{Y}_{T}$ is the column vector of the responses of sample points; 1 is a $1 \times N_{\mathrm{t}}$ unit vector. The prediction value and standard deviation of the unvisited point using the Kriging model are formulated in Eq. (14):

$$
\begin{aligned}
& \hat{f}_{\mathrm{KRG}}(\boldsymbol{x})=\hat{\mu}+\boldsymbol{r}^{\mathrm{T}} \boldsymbol{R}^{-1}\left(\boldsymbol{Y}_{T}-\mathbf{1}^{\mathrm{T}} \hat{\mu}\right) \\
& \hat{s}_{\mathrm{KRG}}(\boldsymbol{x})=\hat{\sigma}^{2}\left[1-\boldsymbol{r}^{\mathrm{T}} \boldsymbol{R}^{-1} \boldsymbol{r}+\frac{\left(1-\mathbf{1}^{\mathrm{T}} \boldsymbol{R}^{-1} \boldsymbol{r}\right)^{2}}{\mathbf{1}^{\mathrm{T}} \boldsymbol{R}^{-1} \mathbf{1}}\right]
\end{aligned}
$$

\subsubsection{Ensemble method}

In general, different metamodels exhibit different approximation performance levels for different problems. To exploit various metamodels, different metamodels can be combined to formulate an ensemble metamodel to improve the approximation capability [47], as shown in Eq. (15):

$$
\hat{f}_{\text {Ensemble }}=\sum_{i=1}^{N_{\text {esm }}} \alpha_{i} \hat{f}_{i}(\boldsymbol{x})
$$

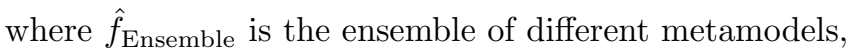
$\hat{f}_{i}(\boldsymbol{x})$ is the $i$ th metamodel, $N_{\text {esm }}$ is the number of different metamodels, and $\alpha_{i}$ is the weight of the corresponding metamodel. In practice, the weights $\left(\alpha_{i}\right)$ can be determined with respect to the approximation error of each metamodel $[47,48]$. If the computational cost is acceptable, the weights can be directly optimized using numerical methods by minimizing the cross-validation error of the ensemble metamodel [43, 49].

\subsubsection{Machine-learning-based metamodeling me- thod}

Because the essence of metamodels in optimization is relatively similar to the regression task in machine learning, some machine learning regression algorithms have been applied to metamodeling in practice. The representative method is support vector regression $[50,51]$. Support vector regression is based on the principle of support vector machines with slight variations and has proven to be effective in solving regression problems [51]. Artificial neural networks are also common in metamodeling fields [52]. An artificial neural network is a multilayer feedforward network that approximates the targeted nonlinear function; the network parameters are trained via numerical methods (e.g., back-propagation algorithm). As a hierarchical composition of Gaussian process-based metamodels (e.g., Kriging), deep Gaussian processes have attracted considerable interest in recent years owing to their promising nonlinear approximation performance [53-55].

\subsection{Multi-model fusion methods}

Most conventional metamodeling techniques and metamodel-based optimization processes simply utilize high-fidelity analysis models that are accurate but computationally intensive. Thus, the computational burden for solving MDO problems remains heavy because of the excessive number of costly samples. However, real-world aerospace system designs generally involve multi-fidelity analysis models, such as structural FEA models with fine or coarse grids and orbital transfer models considering perturbations and eclipses. Low-fidelity analysis models are less accurate but computationally inexpensive. Multimodel fusion methods (or multi-fidelity methods) have been proposed to exploit multi-fidelity analysis models [56-60]. In this approach, a large number of lowfidelity points are generated to capture the trends of system responses, and a small number of high-fidelity points are used to calibrate the trend. The basic form of a multi-model fusion metamodel is given by Eq. (16). In terms of the method for determining the regression $(\rho)$ and discrepancy $(\hat{\delta}(\boldsymbol{x}))$ items, multi-model fusion methods can be divided into correction-based and Bayesian methods.

$$
f_{\mathrm{HF}}(\boldsymbol{x})=\rho \hat{f}_{\mathrm{LF}}(\boldsymbol{x})+\hat{\delta}(\boldsymbol{x})
$$

\subsubsection{Correction-based method}

The correction-based method is a straightforward multimodel fusion method, where the metamodel of the lowfidelity model $\left(\hat{f}_{\mathrm{LF}}(\boldsymbol{x})\right)$ and that of the discrepancy $(\hat{\delta}(\boldsymbol{x}))$ are constructed. The regression item $(\rho)$ is incorporated to minimize the difference between the scaled low-fidelity 
model approximation $\left(\rho \hat{f}_{\mathrm{LF}}(\boldsymbol{x})\right)$ and high-fidelity model response $\left(f_{\mathrm{HF}}(\boldsymbol{x})\right)$. Traditionally, $\rho$ is a scalar, but modeling it as a function of $\boldsymbol{x}$ is an area of research $[61,62]$. Based on Ref. [59], radial basis function and Kriging are the most widely used metamodels in the correction-based multi-model fusion method.

\subsubsection{Bayesian method}

The Bayesian multi-model fusion method, also known as the Co-Kriging method, was introduced by Kennedy and O'Hagan [63]. Co-Kriging constructs the covariance between high-fidelity and low-fidelity models to import the assistance of the low-fidelity model; in this method, $\rho$ and the hyperparameters of $\hat{\delta}(\boldsymbol{x})$ are both estimated. Forrester et al. applied Co-Kriging to the design optimization field by combining it with the Bayesian model update criterion to balance global exploration and local exploitation [56]. In recent decades, Co-Kriging variants have become one of the most popular multi-model fusion methods [59]. Different variants of Co-Kriging have also been proposed to improve the approximation accuracy [64-67] and reduce the metamodeling cost [66-69].

To illustrate the concept of multi-model fusion intuitively, a one-dimensional (1D) numerical example is presented. This example has been widely reported in the literature to demonstrate the effects of multi-model fusion methods $[56,57,59,66,70]$. High-fidelity and low-fidelity models are formulated in Eq. (17). Here, the parameters adopt $A=0.5, B=10$, and $C=-5$. A Kriging model is constructed using pure high-fidelity samples. A Co-Kriging model is constructed using both high-fidelity and low-fidelity samples. As illustrated in Fig. 7, Kriging inadequately approximates $f_{\mathrm{HF}}$, whereas the Co-Kriging calculation is relatively close to $f_{\mathrm{HF}}$ with the support of low-fidelity data.

$$
\left\{\begin{array}{l}
f_{\mathrm{HF}}(x)=(6 x-2)^{2} \sin (12 x-4) \\
f_{\mathrm{LF}}(x)=A f_{\mathrm{HF}}+B(x-0.5)+C
\end{array}, x \in[0,1]\right.
$$

\section{Metamodel-based multidisciplinary optimization strategy}

\subsection{Infill sampling criterion-based optimiza- tion strategy}

In the dynamic metamodel-based optimization strategy, one widely used method for updating metamodels is to sequentially allocate the newly added sample points in

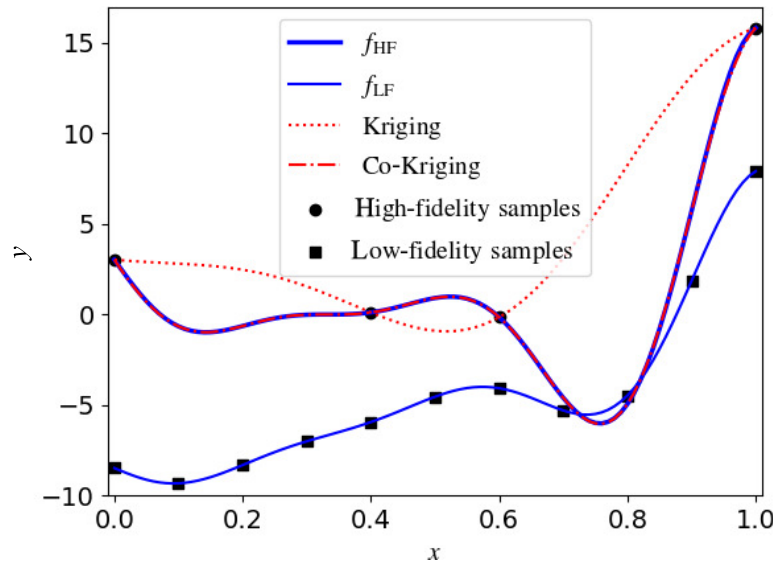

Fig. 7 1D numerical example for Co-Kriging.

the design space according to a certain infill criterion. In this study, two representative infill sampling criterionbased optimization strategies are introduced: efficient global optimization methods and mode-pursing sampling methods.

\subsubsection{Efficient global optimization method}

The well-known efficient global optimization algorithm generates infill sample points in the design space [71], where the expected improvement is maximized to balance the exploration and exploitation of optimization. The formulation of the expected improvement is given by Eq. (18):

$$
\begin{aligned}
& E I(\boldsymbol{x})=E[I(\boldsymbol{x})] \\
& = \begin{cases}\left(y_{\mathrm{min}}-\hat{f}_{\mathrm{KRG}}(\boldsymbol{x})\right) \Phi\left(\frac{y_{\mathrm{min}}-\hat{f}_{\mathrm{KRG}}(\boldsymbol{x})}{\hat{s}_{\mathrm{KRG}}(\boldsymbol{x})}\right) \\
+\hat{s}_{\mathrm{KRG}}(\boldsymbol{x}) \phi\left(\frac{y_{\mathrm{min}}-\hat{f}_{\mathrm{KRG}}(\boldsymbol{x})}{\hat{s}_{\mathrm{KRG}}(\boldsymbol{x})}\right), & \hat{s}_{\mathrm{KRG}}(\boldsymbol{x})>0 \\
0, & \hat{s}_{\mathrm{KRG}}(\boldsymbol{x})=0\end{cases}
\end{aligned}
$$

where $\Phi(\cdot)$ and $\phi(\cdot)$ are the Gaussian probability distribution function and probability density function, respectively; $y_{\min }$ is the minimum objective function value among existing sample points. As shown in Eq. (18), if the newly added infill sample point is not the same as existing sample points, then $E I(\boldsymbol{x})>0$; otherwise, $E I(\boldsymbol{x})=0$.

To illustrate the efficient global optimization process intuitively, a 1D numerical example (Eq. (19)) is investigated.

$$
f(x)=(6 x-2)^{2} \sin (12 x-4)
$$

The optimization process is illustrated in Fig. 8. The figure shows that the sample point with the maximum 


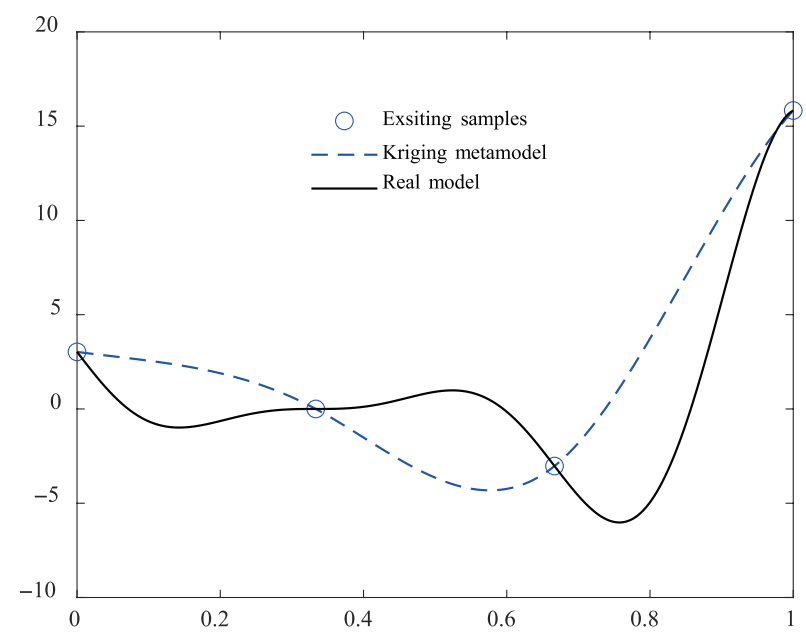

(a) Initial Kriging model

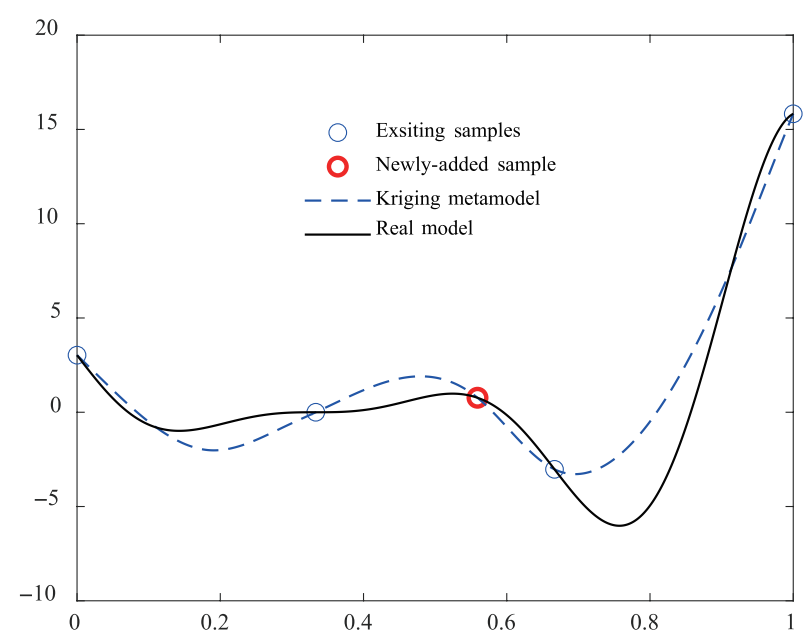

(c) Kriging model after the 1st iteration

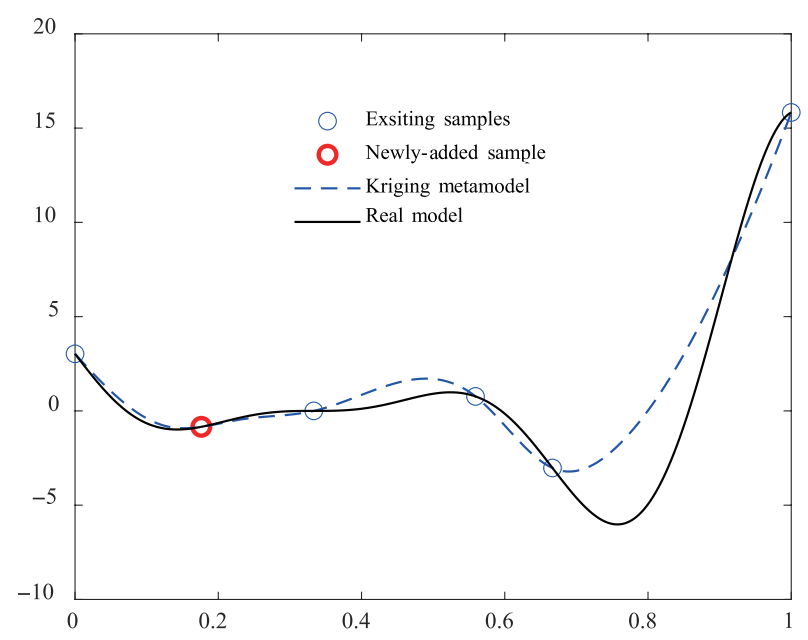

(e) Kriging model after the 2nd iteration

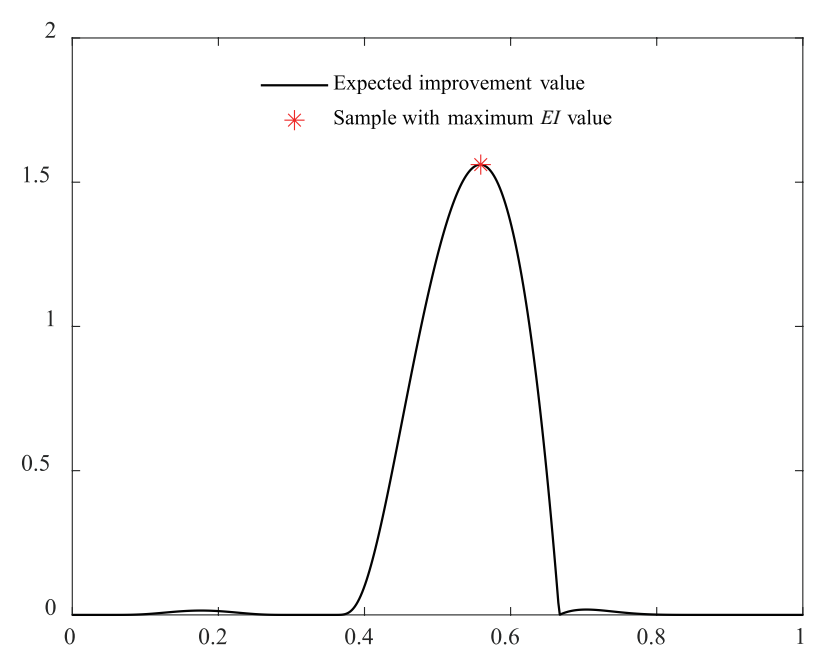

(b) Initial $E I(\boldsymbol{x})$ curve

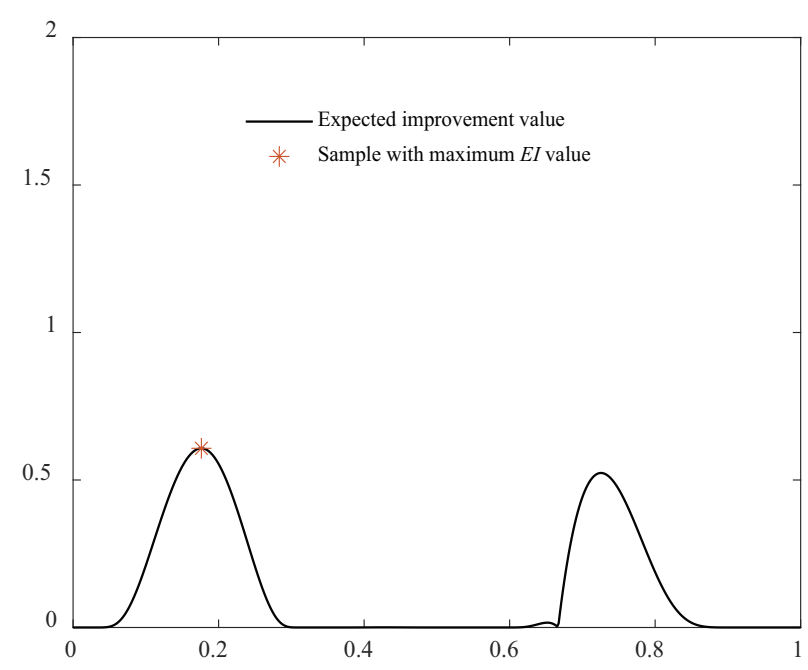

(d) $E I(\boldsymbol{x})$ after the 1st iteration

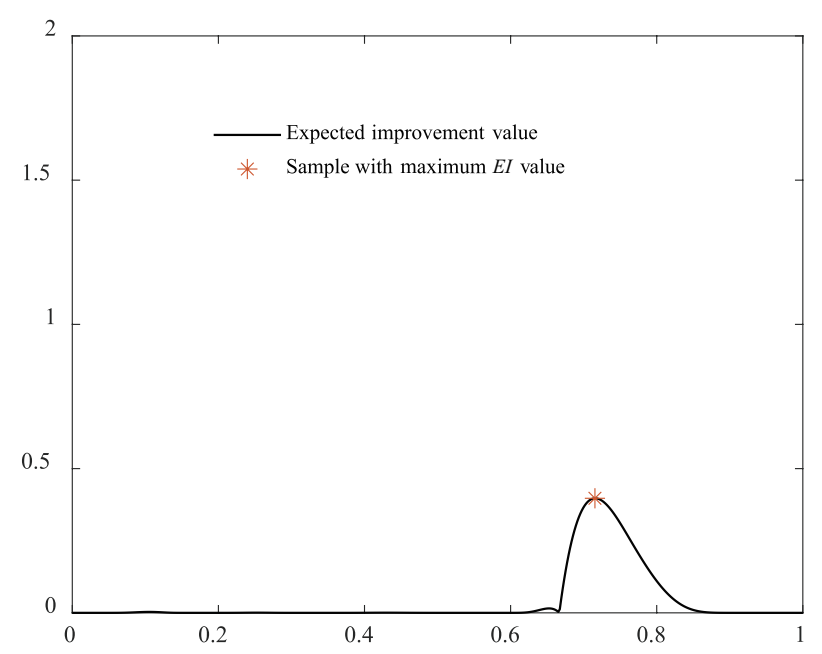

(f) $E I(\boldsymbol{x})$ after the 2nd iteration

Fig. 8 Efficient global optimization process. 


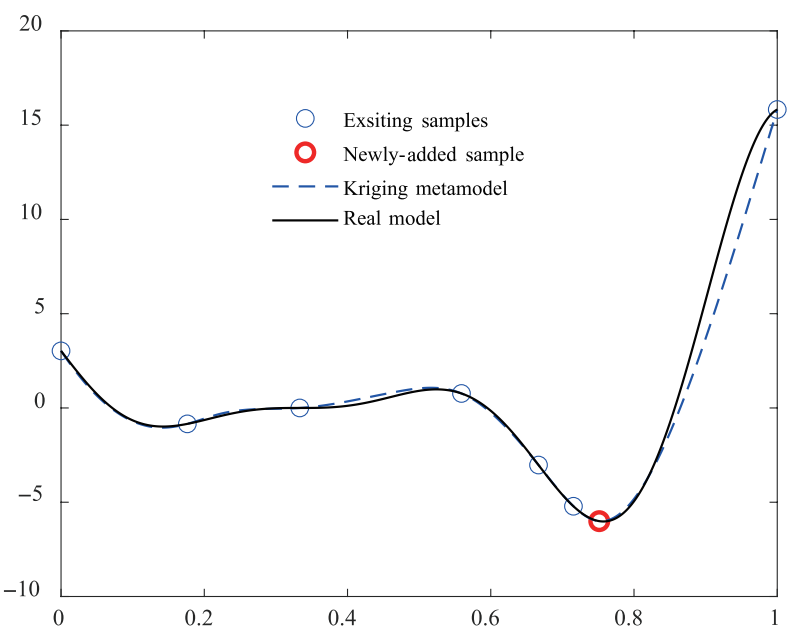

(g) Kriging model after the 3rd iteration

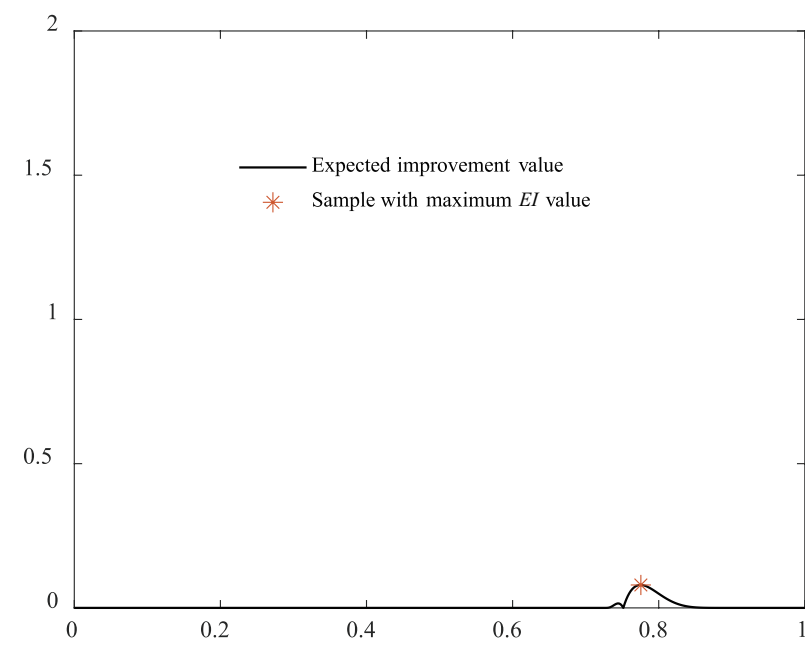

(h) $E I(\boldsymbol{x})$ after the 3rd iteration

Fig. 8 Efficient global optimization process. (Continued)

$E I(\boldsymbol{x})$ in each iteration is selected to update the Kriging model. In addition, $E I(\boldsymbol{x})$ decreases with iteration, indicating the convergence tendency of the optimization process. After the 3rd iteration, the optimization process converges to the global optimum.

To further improve the optimization capacity, some variants of efficient global optimization have been developed in recent years, especially in constraint handling [72], parallel infill sampling [73], and highdimensional optimization [74]. Further details of the variants of efficient global optimization are discussed in Refs. [75-78].

\subsubsection{Mode-pursing sampling methods}

The mode-pursing sampling method proposed by Wang et al. is another typical infill-criterion-based optimization strategy, where the infill criterion is implemented based on a constructed cumulative probability function [79]. In the traditional mode-pursing sampling method, the biased cumulative sum of approximated objective values of numerous inexpensive points constructs the cumulative distribution function, as given in Eq. (20):

$$
\begin{aligned}
& \hat{G}(i)=G(i)^{1 / r\left(R^{2}\right)}, i=1, \cdots, N_{\mathrm{cg}} \\
& G(i)=\sum_{k=1}^{i} \sum_{j=(k-1) \cdot n_{\mathrm{g}}+1}^{k \cdot n_{\mathrm{g}}}\left(f_{\max }-\hat{f}\left(\boldsymbol{x}_{j}\right)\right) / n_{\mathrm{g}} \\
& r\left(R^{2}\right)= \\
& \begin{cases}1, & R^{2}<0.8 \\
\frac{\log G_{\min }}{\log 0.75} \cdot\left[1+\sqrt{1-\frac{\left(R^{2}-0.8\right)^{2}}{0.2^{2}}}\right], & 0.8 \leqslant R^{2} \leqslant 1\end{cases}
\end{aligned}
$$

where $\hat{G}(i)$ is the cumulative distribution function, $G(i)$ is the cumulative sum, $G_{\min }$ is the minimum cumulative sum, $r\left(R^{2}\right)$ is the bias control factor determined by the complex correlation coefficient $\left(R^{2}\right)$ of the metamodel, $N_{\text {cg }}$ is the number of inexpensive point groups, and $n_{\mathrm{g}}$ is the number of each group.

The optimization process of mode-pursing sampling is illustrated in Fig. 9 [79], using a six-hump camel-back problem [80], as formulated in Eq. (21):

$$
\begin{aligned}
f(\boldsymbol{x})= & 4\left(x^{(1)}\right)^{2}-\frac{21}{10}\left(x^{(1)}\right)^{4}+\frac{1}{3}\left(x^{(1)}\right)^{6}+x^{(1)} x^{(2)} \\
& -4\left(x^{(2)}\right)^{2}+4\left(x^{(2)}\right)^{4} ; \boldsymbol{x} \in[-2,2]^{2}
\end{aligned}
$$

(a)

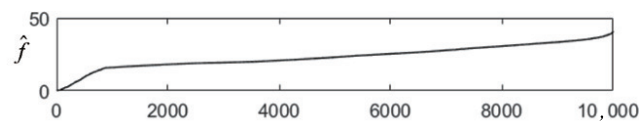

(b)

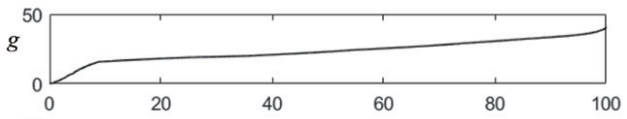

(c)

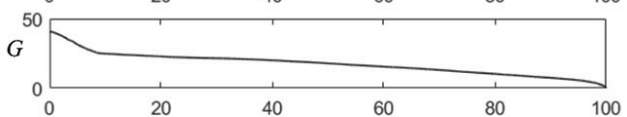

(d) $\hat{G}$

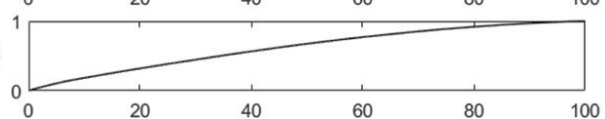

(e)

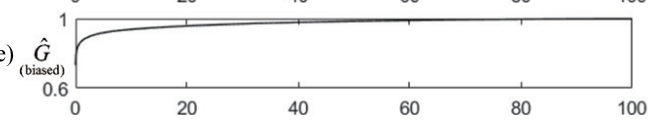

Fig. 9 Mode-pursing sampling optimization procedure.

In the mode-pursing sampling procedure, numerous inexpensive points are randomly generated in the design space and sorted in ascending order with respect to their 
metamodel responses, $\hat{f}\left(\boldsymbol{x}_{i}\right)$, as shown in Fig. 9(a). Then, $\hat{f}\left(\boldsymbol{x}_{i}\right)$ is subtracted from the maximum $\hat{f}\left(\boldsymbol{x}_{i}\right)$ to obtain a nonnegative difference (Fig. 9(b)), which is averaged over a group of $n_{\mathrm{g}}$ inexpensive points to obtain $G(i)$ (Fig. 9(c)). Then, $G(i)$ is normalized as $\hat{G}\left(x_{i}\right)$, as shown in Fig. 9(d). Here, $\hat{G}(i)$ satisfies the requisites of a cumulative distribution function, such as that monotonically increasing and varying between 0 and 1 . New sample points are selected from inexpensive points based on $\hat{G}(i)$, and the selected probability of inexpensive points in the $i$ th group is equal to $\hat{G}(i)$. The control factor, $r\left(R^{2}\right)$, biases $\hat{G}(i)$ toward the inexpensive point groups with smaller $\hat{f}\left(\boldsymbol{x}_{i}\right)$ values, as illustrated in Fig. 9(e). In other words, the more accurate the metamodel, the more likely inexpensive points $\left(\hat{f}\left(\boldsymbol{x}_{i}\right)\right)$ are selected. Because $\hat{G}(i)$ is positive throughout the design space, and mode-pursing sampling adopts an elite strategy, the global convergence property can be theoretically proved easily $[79,81]$.

Because of the potential global optimization performance of the mode-pursing sampling framework, a range of variants has been proposed for different optimization problems by customizing the expression or application scenario of $\hat{G}(i)$. For instance, Kazemi et al. proposed the constraint-importance mode-pursuing sampling method for constrained optimization problems by introducing penalty items into $\hat{G}(i)$ [82]. Sharif et al. developed discrete variable mode-pursing sampling for discrete variable optimization problems by replacing a continuous objective with a discrete objective and utilizing a doublesphere strategy to improve the local exploitation ability [83]. Shan and Wang proposed a Pareto set pursing method for multi-objective optimization problems by aggregating multiple objectives into a single fitness function that reflects the dominance of sample points [84]. Other variants have also been developed for high-dimensional expensive black-box optimization problems $[81,85]$ or high-dimensional expensive constrained black-box optimization problems [86].

In addition to the foregoing algorithms, other widely used infill sampling criteria include the minimization of surrogate prediction $[87,88]$, probability of improvement $[89,90]$, mean square error [91, 92], lower confidence bounding [87-95], and fuzzy clustering-based criterion $[96,97]$. Among the different infill sampling criteria, expected improvement, probability of improvement, mean square error, and lower confidence bounding are generally combined with Kriging or other metamodels with error estimation capability $[98,99]$. Moreover, as an ensemble, all criteria can be used to improve robustness; this is called a multi-infill strategy $[97,100,101]$.

\subsection{Space reduction-based optimization strategy}

The space reduction-based optimization strategy is another widely used approach in practice. In this approach, a small subspace in the design space is constructed or identified. This subspace is referred to as the region of interest, where the global optimum is located with high probability. During optimization, a number of newly added samples are generated in the region to gradually improve the fitting quality of metamodels in the vicinity of the global optimum. In general, the space reductionbased optimization strategy includes three major steps: (1) determining the center of the region; (2) calculating the size of the region; and (3) trimming the region according to the design space. The region is identified, as illustrated in Fig. 10. In this paper, several representative identification methods for the ROI are introduced as follows.

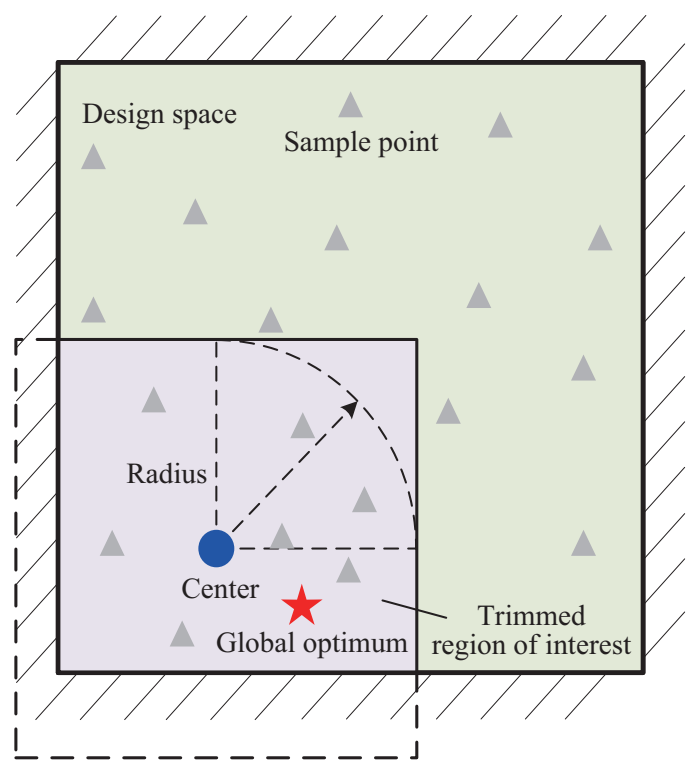

Fig. 10 Space reduction-based optimization strategy.

\subsubsection{Significant design space method}

The significant design space method is a simple spacereduction method [94]. In this method, the region of interest is constructed around the best sample $\left(\boldsymbol{x}_{k}^{*}\right)$ at the $k$ th iteration. The space size $\left(\boldsymbol{B}_{k}\right)$ is determined according to the metamodel approximation accuracy in the vicinity of $\boldsymbol{x}_{k}^{*}$, as shown in Eq. (22): 


$$
\left\{\begin{array}{l}
e_{k}=\left|\left(f_{k}^{*}-\hat{f}_{k}^{*}\right) / f_{k}^{*}\right| \\
\vartheta_{k}=e_{\mathrm{a}} / e_{k}
\end{array}\right.
$$

where $e_{k}$ is the metamodel approximation accuracy in the vicinity of $\boldsymbol{x}_{k}^{*} ; f_{k}^{*}$ and $\hat{f}_{k}^{*}$ are the objective function and metamodel prediction values at $\boldsymbol{x}_{k}^{*}$, respectively; $\vartheta_{k}$ is the size scale indicator; and $e_{\mathrm{a}}$ is the acceptable deviation typically in the range of 0.001-0.050. Based on Eq. (22), if the metamodel approximation accuracy is satisfied (i.e., $\left.\vartheta_{k}>1\right)$, then $\boldsymbol{B}_{k}$ is increased to $\boldsymbol{B}_{k-1} / \alpha$ to explore the global optimum; otherwise, $\boldsymbol{B}_{k}$ is decreased to $\boldsymbol{B}_{k-1} \cdot \alpha$ to improve the metamodel approximation accuracy and exploit the local optimum. If the length in any dimension is no less than the threshold, then the $k$ th trial significant design space is defined as $\boldsymbol{S}_{k}^{\mathrm{t}}=\left[\boldsymbol{B}_{k}^{(\mathrm{L})}, \boldsymbol{B}_{k}^{(\mathrm{U})}\right]$, as shown in Eq. (23):

$$
\begin{aligned}
\boldsymbol{B}_{k}^{(\mathrm{L})} & =\boldsymbol{x}_{\text {best }}-\boldsymbol{B}_{k} \\
\boldsymbol{B}_{k}^{(\mathrm{U})} & =\boldsymbol{x}_{\text {best }}+\boldsymbol{B}_{k}
\end{aligned}
$$

To prevent the significant design space from exceeding the entire design space, $\boldsymbol{S}_{0}$, the $k$ th space, $\boldsymbol{S}_{k}$, is defined as the intersection between $\boldsymbol{S}_{k}^{\mathrm{t}}$ and $\boldsymbol{S}_{0}$, as illustrated in Fig. 10.

\subsubsection{Trust region method}

The trust region method is a widely used space reduction method for refining metamodels, where the size of the region of interest is adjusted according to the prediction performance of the objective improvement [102], as shown in Eq. (24):

$$
\Delta f=\left(f_{k-1}^{*}-f_{k}^{*}\right) /\left(f_{k-1}^{*}-\hat{f}_{k}^{*}\right)
$$

where $\Delta f$ is the prediction performance of objective improvement. In Eq. (24), a larger $\Delta f$ indicates that the optimization process can lead to a better optimum. A smaller $\Delta f$ indicates that the improvement in the objective is insignificant. Note that the objective cannot be improved if $t$ is negative.

The size of the region can be determined in terms of the radius of the trust region, as shown in Eq. (25):

$$
\delta_{k+1}= \begin{cases}c_{1}\left\|\boldsymbol{x}_{k}^{*}-\boldsymbol{x}_{k-1}^{*}\right\|, & \Delta f<0.1 \\ \min \left(c_{2}\left\|\boldsymbol{x}_{k}^{*}-\boldsymbol{x}_{k-1}^{*}\right\|, \Delta\right), & \Delta f>0.75 \\ \left\|\boldsymbol{x}_{k}^{*}-\boldsymbol{x}_{k-1}^{*}\right\|, & 0.10 \leqslant \Delta f \leqslant 0.075 \\ \multicolumn{2}{c}{0<c_{1}<1, c_{2}>1}\end{cases}
$$

where $\delta_{k+1}$ is the radius of the trust region at the $(k+1)$ th iteration; $\boldsymbol{x}_{k}^{*}$ and $\boldsymbol{x}_{k-1}^{*}$ are the best sample points at the $k$ th and $(k-1)$ th iterations, respectively; $c_{1}$ and $c_{2}$ are the shrinking and amplification factors, respectively; and $\Delta$ is the upper bound of the trust region radius. As proved in Ref. [102], the trust region-based optimization strategy can converge to the local optimum, which can also converge to the global optimum. In recent years, some trust region method-based adaptive optimization methods have been developed to further improve the optimization performance, as detailed in Refs. [103-105].

\subsubsection{Interesting sampling region method}

The interesting sampling region method is a machinelearning-assisted method for locating the potential region containing the global optimum [106]. In this method, the existing samples are classified into two categories with respect to their responses. If the objective value at the sample point is less than the predefined threshold, $P_{\text {thresh }}$, the sample is labeled as a superior sample, indicating that the sample is probably located close to the global optimum. A binary machine learning classifier (e.g., support vector machine [107] and Bayes classifier [108]) is constructed based on labeled samples. Then, a larger number of inexpensive geometry points are generated via Latin hypercube design in the design space in which superior inexpensive points are identified by the trained classifier. The cluster center of superior inexpensive points is determined to depict the potential location of the global optimum. For instance, consider the six camelhump functions; the superior inexpensive points (i.e., dots labeled with red circles) under different classification thresholds $\left(P_{\text {thresh }}\right)$ are illustrated in Fig. 11. The figure shows that the potential location can be depicted by the cluster of superior inexpensive points using a proper $P_{\text {thresh value. }}$

Finally, the interesting sampling region at the $k$ th iteration is defined as a hypercube subregion, as shown in Eq. (26):

$$
\begin{aligned}
& \boldsymbol{I S} \boldsymbol{R}^{(k)}=\left[\boldsymbol{x} \mid \boldsymbol{x}_{\mathrm{ISR}}^{(k)}-\boldsymbol{R}^{(k)} \leqslant \boldsymbol{x} \leqslant \boldsymbol{x}_{\mathrm{ISR}}^{(k)}+\boldsymbol{R}^{(k)}\right] \\
& \text { where } \quad \boldsymbol{R}^{(k)}=\eta\left\|\boldsymbol{x}_{\mathrm{pse}}^{*}-\boldsymbol{x}_{\mathrm{ISR}}^{(k)}\right\|_{2}
\end{aligned}
$$

where the current pseudo-optimum $\left(\boldsymbol{x}_{\text {ISR }}^{(k)}\right)$ is the center of the region; $\boldsymbol{x}_{\mathrm{pse}}^{*}$ is the cluster center of superior inexpensive points (also regarded as the potential position of global optimum); $\boldsymbol{R}^{(k)}$ is the Euclidean distance between $\boldsymbol{x}_{\mathrm{ISR}}^{(k)}$ and $\boldsymbol{x}_{\mathrm{pse}}^{*}$; and $\eta$ is the shrinking coefficient used to adjust the size of $\boldsymbol{R}^{(k)}$. As the optimization proceeds, the interesting sampling region is dynamically scaled based on the Euclidean distance between $\boldsymbol{x}_{\mathrm{ISR}}^{(k)}$ and $\boldsymbol{x}_{\mathrm{pse}}^{*}$, 

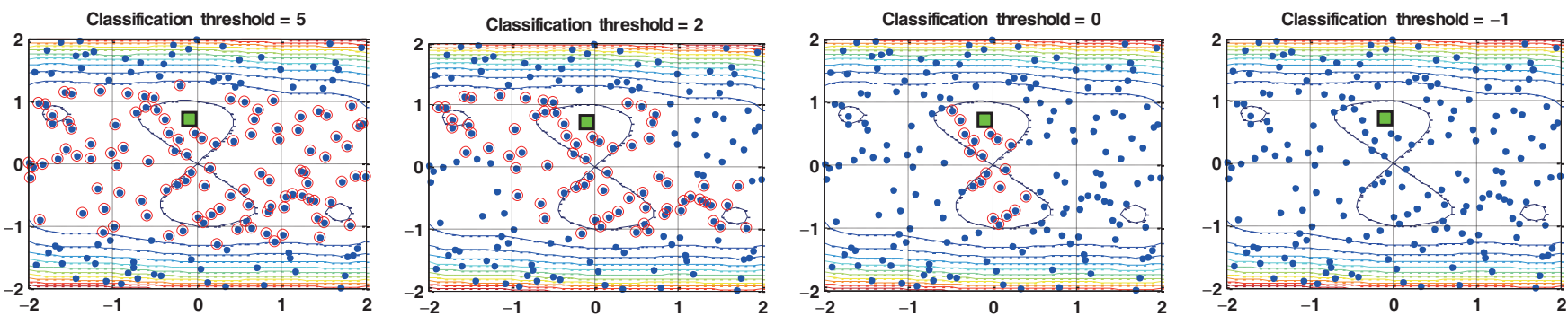

Fig. 11 Identification of superior inexpensive points.

considerably improving the optimization efficiency and global convergence. Once the current pseudo-optimum $\left(\boldsymbol{x}_{\text {ISR }}^{(k)}\right)$ is far from the potential global optimum (i.e., $\left.\left\|\boldsymbol{x}_{\mathrm{pse}}^{*}-\boldsymbol{x}_{\mathrm{ISR}}^{(k)}\right\|_{2} \geqslant\left\|\boldsymbol{x}_{\mathrm{pse}}^{*}-\boldsymbol{x}_{\mathrm{ISR}}^{(k-1)}\right\|_{2}\right)$, the exploration region must be enlarged to include areas that may not have been covered, thus avoiding missing the true optimum. Additionally, if $\boldsymbol{x}_{\mathrm{ISR}}^{(k)}$ is probably in the vicinity of the global optimum, then the search region can be reduced to improve the approximation accuracy of the constructed metamodels.

In addition to the aforementioned methods, many other space reduction methods have been developed. For instance, Wang et al. proposed an adaptive response surface method to solve computation-intensive design problems, where regions with inferior sample points are cut for sampling in the reduced design space and updating the metamodels [109]. In addition, some variants of the adaptive response surface method were investigated to further improve optimization convergence and robustness [110-112]. Dong et al. proposed a new space reduction-based optimization algorithm to solve the unconstrained expensive black-box optimization problems, where a score-based reduced subspace around the current best sample is created to accelerate the local convergence [113]. Qiu et al. proposed self-organizing maps and fuzzy clustering-based three-stage space reduction and metamodeling optimization methods to improve efficiency and robustness performance [114]. Liu et al. developed a Monte Carlo method and space reduction strategy to improve the efficiency of the sampling process [115].

\subsection{Surrogate-assisted evolutionary algo- rithms}

Motivated by the idea of merging surrogates into the evolutionary process, surrogate-assisted evolutionary algorithms have received considerable attention in recent years $[116,117]$. In the optimization of surrogate-assisted evolutionary algorithms, metamodels are developed as "surrogates" of expensive simulation models for stochastic evolutionary operations. This can effectively alleviate the computational complexity in engineering optimization. Several potential solutions (called individuals or particles) are also concurrently selected as newly added sample points to improve the surrogate approximation accuracy in the vicinity of the optimum. The fundamental architecture of the algorithm is graphically illustrated in Fig. 12

The selection of newly added infill sample points from the offspring population is found to be a crucial step in the algorithm [116]. The commonly used selection criteria can be generally divided into three categories: performancebased, uncertainty-based, and hybrid criteria [118]. In the performance-based criterion, the infill sample points are selected by considering only the predicted fitness values $[117,119,120]$. Generally, this criterion cannot be exclusively used because optimization probably falls into the local optima once the surrogates fail to approximate the simulation model well. The uncertainty-based criterion chooses infill sample points with considerable uncertainties, effectively improving the accuracy of surrogates and leading the search to unexplored regions [121]. However, numerous simulation model evaluations are performed to explore regions with sparse samples, resulting in a low convergence rate [118]. The hybrid criterion simultaneously incorporates performance and uncertainty $[119,122]$. The primary objective of this criterion is to balance the surrogate approximation accuracy and population competitiveness during optimization.

In recent years, a number of surrogate-assisted evolutionary algorithms have been successfully applied to solve various optimization problems, such as unconstrained/constrained [119,123-130], multi-objective [131137], and multi-fidelity optimization problems $[138,139]$. 


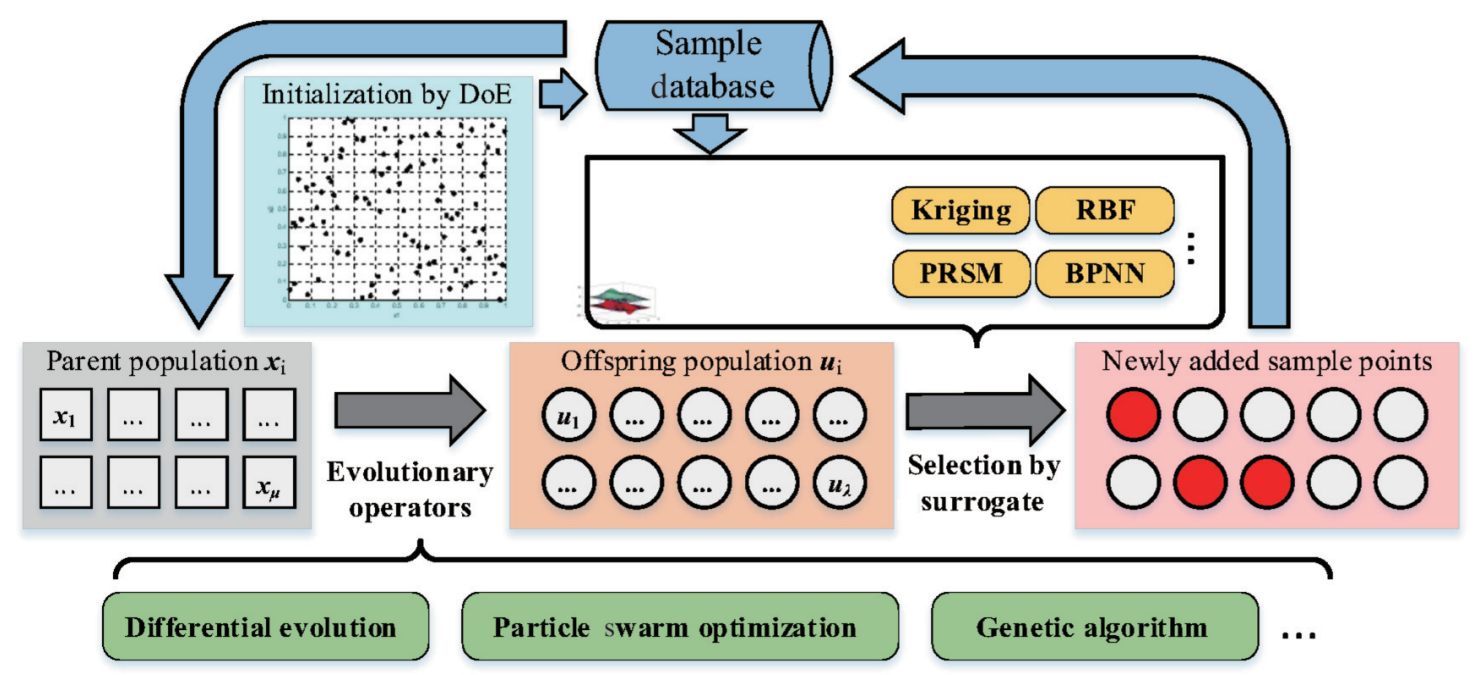

Fig. 12 Optimization mechanism of surrogate-assisted evolutionary algorithms.

\subsection{Metamodel-based optimization en- hanced by machine learning}

Machine learning is a well-known state-of-the-art technology that has been successfully applied to big data mining, natural language processing, image recognition, medical diagnosis, and so on. Similarly, a considerable amount of research has been conducted to integrate machine learning techniques (e.g., classification learning, cluster analysis, and dimensionality reduction) into engineering optimization. Classification learning is among the most widely used machine learning techniques in optimization and has considerable potential for identifying the feasibility of a solution during the optimization process. Motivated by this concept, several studies have been performed to improve the optimization performance by employing a support vector machine or its variants for identifying superior inexpensive points [140-144]. Additionally, other classification learning techniques, such as decision tree [145] and k-nearest neighbor [146], are employed for optimization. To capture the potential regions effectively, classified points are further categorized based on a set of user-defined characteristics or attributes using cluster analysis techniques. For instance, cluster analysis can be combined with a support vector machine to identify a potential search region by clustering numerous superior inexpensive points, as described in Refs. [21,106]. In multi-objective optimization, cluster analysis can also be implemented to improve the spatial uniformity of the Pareto frontier when determining newly infilled sample points or reference vectors $[135,136,147]$.
To alleviate the computational complexity caused by the "curse of dimensionality", the dimensionality reduction technique provides a promising approach for MBDO to solve high-dimensional optimization problems. One typical approach is to conduct sensitivity analysis before optimization starts. Here, primary design variables are reserved for optimization, whereas minor ones are neglected and set as fixed values during optimization [148]. Although the dimensionality of the optimization problem is reduced by sensitivity analysis, the optimality of the obtained optimized solution probably decreases owing to the irrelevance of several design variables. Another dimensionality reduction method is the utilization of manifold learning (e.g., principal component analysis and Sammon mapping) to map existing sample points together with alternative infill sample points from high-dimensional space into low-dimensional space. A low-dimensional surrogate is also trained to select infill sample points as optimization proceeds $[149,150]$.

In modern engineering design, because new problems are generally derived from a series of previously solved tasks, the concept of transfer optimization has been recently developed. It is a newly emerged methodology for improving the optimization performance of a new optimization task by mining existing knowledge [151]. Although numerous transfer optimization-based methods have been recently investigated [152-154], only a few studies on MBDO methods using transfer optimization have been reported [155]. Therefore, further improving the optimization performance by integrating the transfer 
optimization technique into MBDO methods is promising and valuable [151].

In addition to the aforementioned data-driven machine learning techniques, physics-informed machine learning techniques, also known as physics-informed neural networks [156,157], have emerged as an alternative to illposed and inverse problems. Fundamental physical laws and domain knowledge are embedded by exploiting observational data $[158,159]$, tailoring neural network architecture for physics constraints $[160,161]$, and/or imposing physics constraints into the loss function $[162,163]$. The physics-informed neural network is expected to outperform existing machine learning methods in partially understood, uncertain, and high-dimensional problems. It is also another potential research topic in the area of metamodel-based MDO.

\section{Expensive black-box constraint-hand- ling mechanism}

In engineering practice, MDO problems in aerospace systems generally involve various constraints, such as the natural frequencies of a structure and end-of-life power in orbit. Note that because most constraints arise from time-consuming black-box simulation models (e.g., structural FEA model), directly calculating the gradient information of the constraints via numerical methods (e.g., finite difference method) to search for the feasible domain is difficult. Thus, in engineering practice, the solution of MDO problems with numerous expensive black-box constraints remains complex. To overcome this obstacle, some constraint-handling mechanisms have been integrated with metamodel-based MDOs to further improve the optimization capacity.

\subsection{Penalty function-based methods}

Penalty function-based methods are convenient approaches that are most commonly used for handling expensive black-box constraints. Here, the objective function is augmented by constraints to cope with penalty factors, as given in Eq. (27):

$$
F(\boldsymbol{X})=f(\boldsymbol{X})+\lambda \sum_{i}^{m} \max \left(g_{i}(\boldsymbol{X}), 0\right)
$$

where $\lambda$ is the penalty factor, and $F(\boldsymbol{X})$ is the augmented objective function. The metamodel of the augmented objective function is established for optimization, and the infeasible sample point is penalized with respect to the penalty factor scale.

Although penalty function methods are conveniently implemented in practice, determining the proper penalty factor is difficult. If the penalty factor is extremely small, the optimization process is virtually unable to converge to the feasible domain. If the penalty factor is extremely large, the penalty part can overlap the objective, rendering convergence difficult. Nevertheless, the penalty function method remains widely employed in MBDO methods. For instance, a predefined penalty function is employed to handle the expensive constraints [164]. This method is further enhanced in Ref. [110], where the penalty factor is automatically adjusted according to the constraint violation value.

\section{$5.2 \quad$ Filter-based methods}

In view of the difficult implementation of penalty function-based methods, penalty-free methods have become popular in recent years; among these latter methods, filter-based methods are the most representative. The concept of a filter was originally proposed by Fletcher [165] and was successfully applied to many penalty-free numerical optimization methods [166-168]. To illustrate the concept of a filter, the constraint violation function of the MDO problem is defined as follows:

$$
h(\boldsymbol{X})=\max \left\{g_{1}(\boldsymbol{X}), g_{2}(\boldsymbol{X}), \cdots, g_{\mathrm{m}}(\boldsymbol{X})\right\}
$$

where $h(\boldsymbol{X})$ denotes the constraint violation function. A positive $h(\boldsymbol{X})$ represents an infeasible sample point, and a larger $h(\boldsymbol{X})$ generally indicates inadequate infeasibility. Based on the definition of the Pareto non-domination set, the concept of the filter is clarified as follows:

Definition 1. If and only if $f\left(\boldsymbol{x}_{i}\right) \leqslant f\left(\boldsymbol{x}_{1}\right) \cap h\left(\boldsymbol{x}_{i}\right) \leqslant$ $h\left(\boldsymbol{x}_{1}\right)$, then sample point $\boldsymbol{x}_{i}$ is considered to dominate another sample point pair, $\boldsymbol{x}_{1}$; otherwise, $\boldsymbol{x}_{i}$ and $\boldsymbol{x}_{1}$ do not dominate each other.

Definition 2. A filter aggregates non-dominated sample points. If a sample point $\left(\boldsymbol{x}_{i}\right)$ is not dominated by any point in the current filter, then it can be included in the filter; otherwise, it is rejected.

According to the above definitions, the construction of a filter basically means the creation of a Pareto front in terms of $f(\boldsymbol{X})$ and $h(\boldsymbol{X})$. A filter in the $f(\boldsymbol{X})-h(\boldsymbol{X})$

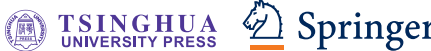


space is illustrated in Fig. 13 [21], where the dots represent the non-dominated sample points. If a newly added sample point is located in the shaded area, then it is not dominated by existing points in the filter, that is, the filter is augmented. If it is located in the hatched area, then the newly added point can dominate at least one point in the filter (i.e., the filter is refined); otherwise, the point is rejected by the filter. In fact, the filter generates a set of blocks for newly added sample points, as indicated by the solid lines in Fig. 13. In constrained optimization procedures, the filter functions as a classifier for accepting or rejecting a sample point, considering both optimality and feasibility. Note that a sample point can be accepted by the filter only if it improves either the optimality or feasibility; hence, the feasibility and optimality of existing sample points are gradually enhanced during the metamodel-based optimization process.

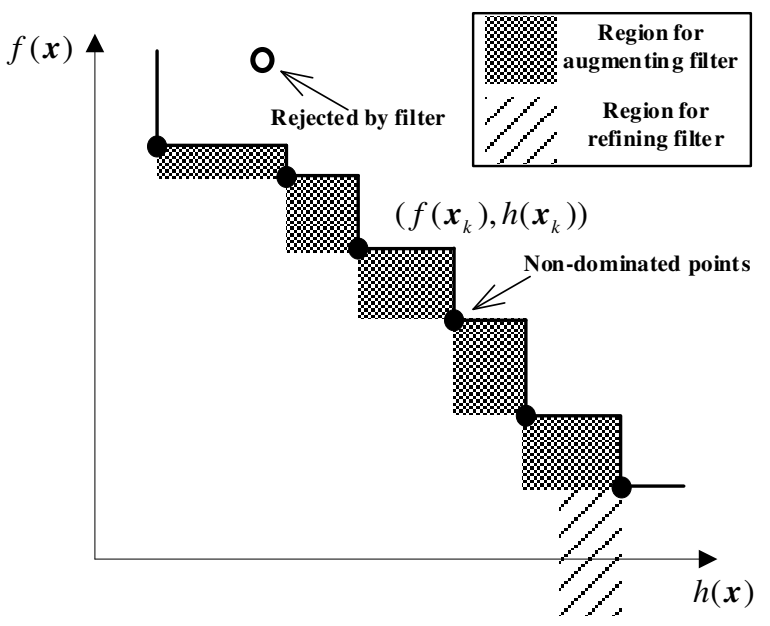

Fig. 13 Filter in $f(\boldsymbol{X})=h(\boldsymbol{X})$ space (reproduced with permission from Ref. [21], (C) The Authors 2018).

A number of MBDO methods have been developed to solve expensive constrained black-box problems based on the filter concept. For instance, the filter technique is combined with a support vector machine to identify a potential sampling sub-region in the vicinity of the global feasible optimum [21]. In Ref. [72], the probability of constrained improvement is enhanced; only the sample point with a positive probability of constrained improvement can be accepted by the filter to enhance optimality and feasibility.

In addition to the aforementioned constraint-handling mechanisms, certain customized methods have also been developed to handle constrained optimization. In
Refs. [16, 169, 170], all constraints are approximated by metamodels to avoid the complicated determination of the penalty factor. Moreover, some sequential updating techniques considering the confidence intervals of Kriging models for constraint approximation were developed $[171,172]$. To further solve problems with numerous constraints, the constraint aggregation method, which lumps numerous constraints into one or a few constraints to significantly reduce the computational cost, has drawn considerable interest in recent years. The KreisselmeierSteinhauser function [173] is a well-known constraint aggregation method that has been used in various applications [86,174,175]. Moreover, based on Refs. [176,177], Kreisselmeier-Steinhauser function-based MBDO methods are efficient in solving expensive black-box constraint problems.

\section{Metamodel-based aerospace system MDO examples}

\subsection{All-electric geostationary Earth orbit satellite MDO problem}

The all-electric geostationary Earth orbit satellite MDO problem is referred from Ref. [19]. The studied allelectric geostationary Earth orbit satellite is a cuboid with a payload module, service module, and solar array. Four Hall-effect thrusters are installed at the bottom of the satellite to execute attitude control, geosynchronous transfer, and geostationary Earth orbit station-keeping maneuvers, as shown in Fig. 14 [19]. The all-electric geostationary Earth orbit satellite MDO problem involves geosynchronous transfer, geostationary keeping, power, thermal control, attitude control, and structure disciplines. The design structure matrix of the MDO problem is shown in Fig. 15 [19], and the involved analysis models of disciplines are detailed in Ref. [19].

The satellite MDO problem was formulated using Eq. (29) [19]. The design variables are steer angles $(\alpha, \beta, \varphi)$ during low-thrust geosynchronous transfer, positions of electric thrusters $\left(d_{\mathrm{T}}, d_{\mathrm{N}}\right)$, battery capacity $\left(C_{\mathrm{s}}\right)$, area of solar arrays $\left(A_{\mathrm{sa}}\right)$, area of thermal radiator $\left(A_{\mathrm{r}}\right)$, angular momentum of reaction wheel $\left(H_{\mathrm{w}}\right)$, and thickness of composite material structure ( $\mathrm{SH}, \mathrm{CH}, \mathrm{TBH}, \mathrm{SP}, \mathrm{CP}, \mathrm{TBP})$. These are optimized to minimize the total mass of the satellite $\left(M_{\text {satellite }}\right)$ subject to the constraints of total transfer time $\left(t_{\mathrm{f}}\right)$, stationkeeping accuracy $\left(\lambda_{\max }\right.$ and $\left.i_{\max }\right)$, available power $\left(P_{\mathrm{BOL}}\right.$ 


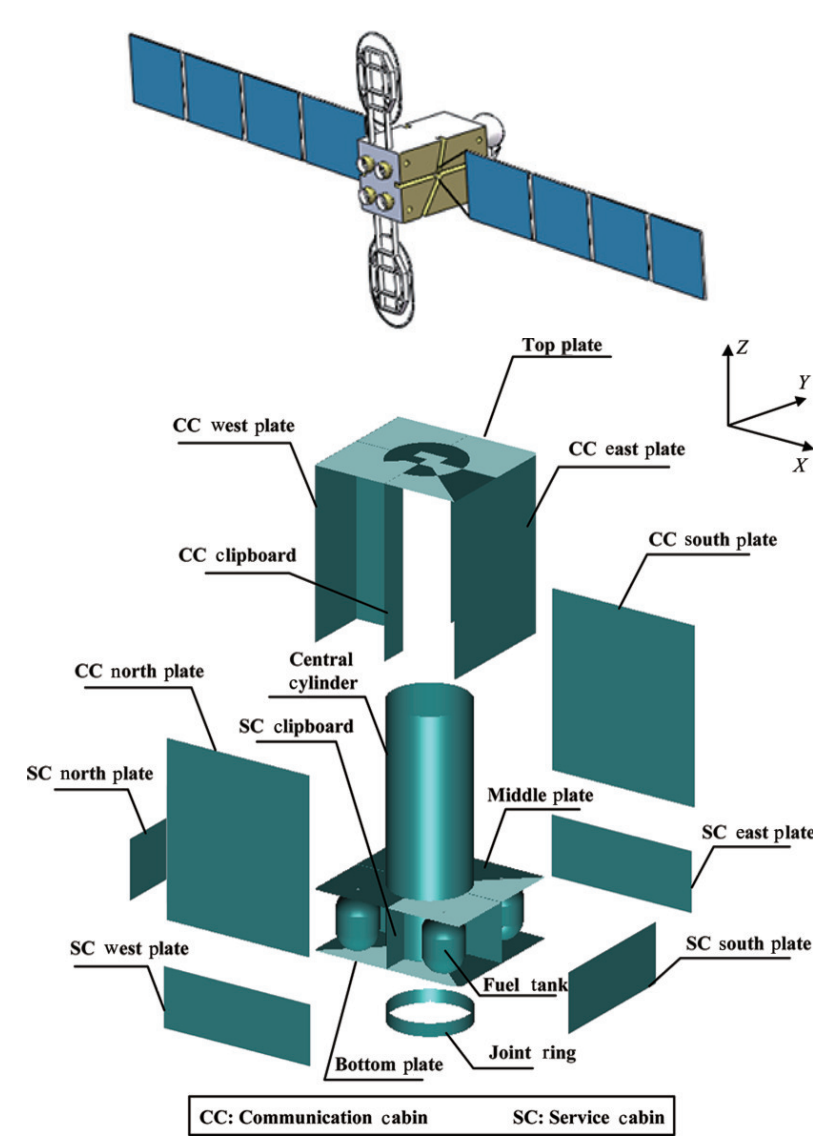

Fig. 14 Schematic of all-electric satellite (reproduced with permission from Ref. [19], (C) IAA 2017).

and $\left.P_{\mathrm{EOL}}\right)$, depth of discharge (DOD), steady-state temperature $(T)$, angular momentum residue $\left(c_{\mathrm{AC}}\right)$, and natural frequencies $\left(f_{X}\right.$ and $\left.f_{Y}\right)$.

$$
\begin{aligned}
\min M_{\text {satellite }}= & m_{\text {payload }}+m_{\text {fuel }}+m_{\text {power }}+m_{\mathrm{TC}} \\
& +m_{\mathrm{AC}}+m_{\text {others }}
\end{aligned}
$$

where $\boldsymbol{X}=\left[\alpha, \beta, \varphi, d_{\mathrm{T}}, d_{\mathrm{N}}, A_{\mathrm{sa}}, C_{\mathrm{s}}, A_{\mathrm{r}}, H_{\mathrm{w}}, \mathrm{SH}, \mathrm{CH}\right.$, $\mathrm{TBH}, \mathrm{SP}, \mathrm{CP}, \mathrm{TBP}]^{\mathrm{T}}$

$$
\text { s.t. }\left\{\begin{array}{l}
t_{\mathrm{f}} \leqslant 180 \text { days, } \lambda_{\max } \leqslant 0.05^{\circ}, i_{\max } \leqslant 0.05^{\circ} \\
P_{\mathrm{BOL}} \geqslant 14.15 \mathrm{~kW}, P_{\mathrm{EOL}} \geqslant 11.90 \mathrm{~kW} \\
267 \mathrm{~K}<T<328 \mathrm{~K}, c_{\mathrm{AC}} \geqslant 0, \mathrm{DOD} \leqslant 0.8 \\
f_{X} \geqslant 14 \mathrm{~Hz}, f_{Y} \geqslant 14 \mathrm{~Hz}
\end{array}\right.
$$

In this example, the filter-based sequential radial basis function method from Ref. [21] was used to solve the MDO problem. The optimization results are summarized in Table 3 [21]. Compared with the conventional genetic algorithm-based multidisciplinary feasible method, the computational cost of the filter-based sequential radial basis function method is reduced by $50 \%$.

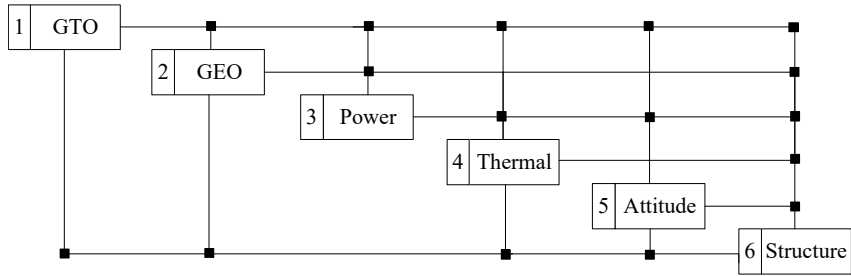

Fig. 15 Design structure matrix of all-electric satellite (reproduced with permission from Ref. [19], C) IAA 2017).

Table 3 Optimization results of design variables

\begin{tabular}{ccc}
\hline Objective & $\begin{array}{c}\text { Genetic } \\
\text { algorithm-based } \\
\text { multidisciplinary } \\
\text { feasible method }\end{array}$ & $\begin{array}{c}\text { Filter-based } \\
\text { sequential radial } \\
\text { basis function } \\
\text { method }\end{array}$ \\
\hline $\begin{array}{c}\text { Total mass }(\mathrm{kg}) \\
\text { Number of } \\
\text { simulations }\end{array}$ & 2396.6 & 2355.9 \\
\hline
\end{tabular}

\subsection{Earth-observation MDO problem}

The Earth observation satellite MDO problem is referred from Ref. [178]. The satellite consists of payload and service cabins, as shown in Fig. 16 [178]. The payloads include a 10-band radiometer and charge-coupled device camera, which are fixed at the bottom of the payload cabin. The solar arrays are set along the south and north faces of the satellite to reduce the influence of external heat flux. Other facilities, such as battery and attitude control subsystems, are placed in the service cabin. The Earth observation satellite MDO problem involves five disciplines: orbit, payload, structure, power, and mass. The design structure matrix of this problem is shown in Fig. 17 [178]. The disciplinary modeling approaches are presented in Ref. [178].

The Earth observation satellite MDO problem also aims to minimize the total mass of the satellite, as formulated in Eq. (30) [178]. The design variables include the aperture and focal length of payloads, area of solar arrays, capacity of storage battery, and thickness of composite material structure. The constraints include the signal-tonoise ratio and resolution of payloads, noise-equivalent temperature difference, surplus power, discharge depth, and natural frequencies.

$$
\begin{aligned}
\min M_{\text {satellite }}= & m_{\text {payload }}+m_{\text {bat }}+m_{\text {solar }} \\
& +m_{\text {str }}+m_{\text {others }}
\end{aligned}
$$

where $\boldsymbol{X}=\left[D_{1}, D_{2}, F_{1}, F_{2}, T_{\mathrm{R}}, T_{\mathrm{BH}}, T_{\mathrm{SH}}, A_{\mathrm{sa}}, C_{\mathrm{s}}\right]^{\mathrm{T}}$ 


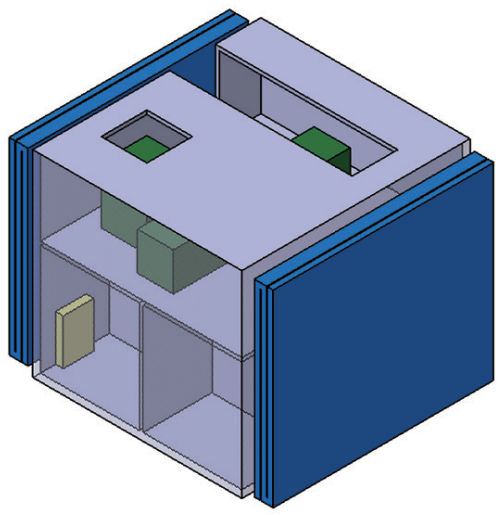

(a)

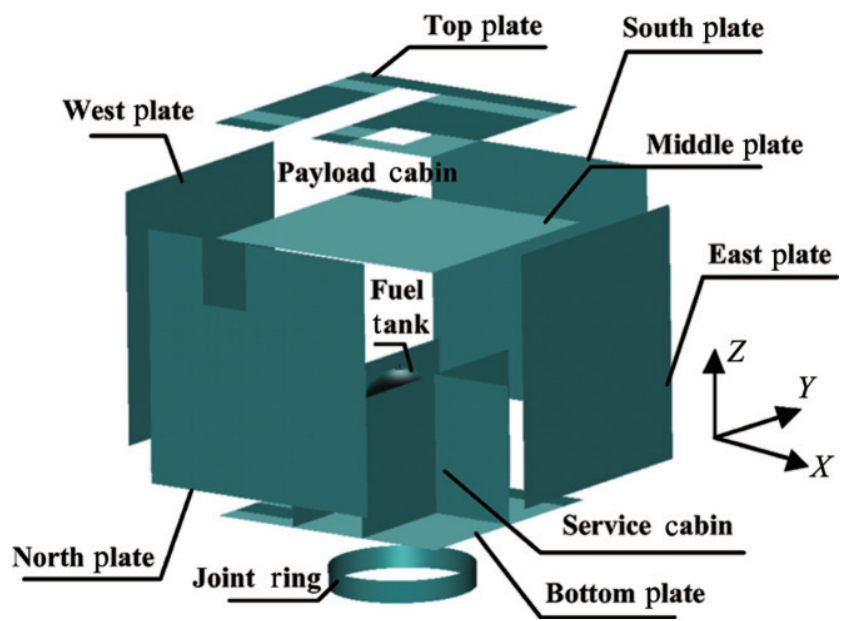

(b)

Fig. 16 Schematic of Earth observation satellite (reproduced with permission from Ref. [178], (C) Taylor \& Francis 2015).

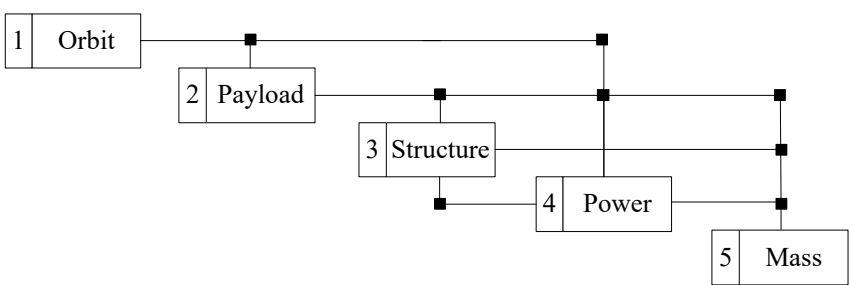

Fig. 17 Design structure matrix of Earth observation satellite MDO problem (reproduced with permission from Ref. [178], C) Taylor \& Francis 2015).

$$
\text { s.t. }\left\{\begin{array}{l}
R_{1} \leqslant 1100 \mathrm{~m}, R_{2} \leqslant 250 \mathrm{~m} \\
\mathrm{SNR}_{1} \geqslant 300, \mathrm{SNR}_{2} \geqslant 500 \\
\quad N E \Delta T \leqslant 0.2 \mathrm{~K} \\
g_{\mathrm{w}} \geqslant 0, \mathrm{DOD} \leqslant 25 \%, f_{x} \geqslant 20 \mathrm{~Hz}, \\
f_{y} \geqslant 20 \mathrm{~Hz}, f_{z} \geqslant 50 \mathrm{~Hz}
\end{array}\right.
$$

In this example, the sequential radial basis function using a support vector machine from Ref. [106] was used to solve the MDO problem. The optimization results are listed in Table 4 [106]. The computational cost of the
Table 4 Comparison of optimization results

\begin{tabular}{ccc}
\hline $\begin{array}{c}\text { Optimization } \\
\text { method }\end{array}$ & $\begin{array}{c}\text { Total mass after } \\
\text { optimization }(\mathrm{kg})\end{array}$ & $\begin{array}{c}\text { Number of } \\
\text { analysis model } \\
\text { evaluations }\end{array}$ \\
\hline $\begin{array}{c}\text { Sequential radial } \\
\text { basis function } \\
\text { using support }\end{array}$ & 340.11 & 165 \\
$\begin{array}{c}\text { vector machine } \\
\text { Genetic algorithm } \\
\text { Sequential } \\
\text { quadratic }\end{array}$ & 354.97 & 7329 \\
programming & 346.15 & 322 \\
\hline
\end{tabular}

sequential radial basis function using the support vector machine is only found to be $2.25 \%$ and $51.24 \%$ of the computational costs of genetic algorithm and sequential quadratic programming, respectively.

\subsection{Satellite constellation MDO problem}

The satellite constellation MDO problem is obtained from Ref. [20], where a small satellite constellation is established to achieve a cooperative Earth observation mission. The design structure matrix of the satellite constellation MDO problem is shown in Fig. 18 [20]. The inter-coupled relationships between the constellation configuration and design of satellite subsystems are considered to enhance system performance. In this example, the constellation is based on the Walker- $\delta$ constellation. In this constellation, the ascending nodes of the orbit planes are uniformly distributed around the equator plane, and the satellites are uniformly distributed within the orbital planes at the same inclination [20].

$$
\begin{aligned}
\min M_{\text {system }}= & \left(m_{\text {payload }}+m_{\text {power }}+m_{\text {thermal }}\right. \\
& \left.+m_{\text {structure }}+m_{\text {others }}\right) \times P \times S
\end{aligned}
$$

where $\boldsymbol{X}_{\mathrm{c}}=\left[h, i, \Omega_{0}, D_{\mathrm{P}}, f_{\mathrm{P}}, A_{\mathrm{s}}, C_{\mathrm{s}}, T_{\mathrm{H}}, T_{\mathrm{P}}\right], \boldsymbol{X}_{\mathrm{d}}=[P, S]$

$$
\text { s.t. }\left\{\begin{array}{l}
C_{\mathrm{R}} \geqslant 0.8 \\
R_{\mathrm{P}} \leqslant 250 \mathrm{~m}, S N R \geqslant 500 \\
\mathrm{DOD} \leqslant 0.3, g_{\mathrm{w}} \geqslant 0 \\
T_{0} \leqslant 303.15 \mathrm{~K} \\
f_{X} \geqslant 20 \mathrm{~Hz}, f_{Y} \geqslant 20 \mathrm{~Hz}
\end{array}\right.
$$

The constellation MDO problem was formulated using Eq. (31). This is a continuous-discrete mixed optimization problem. The continuous design variables $\left(\boldsymbol{X}_{\mathrm{c}}\right)$ for constellation configuration include the orbital altitude $(h)$, inclination $(i)$, and right ascension of the ascending node $(\Omega)$; those for satellite subsystems include the 


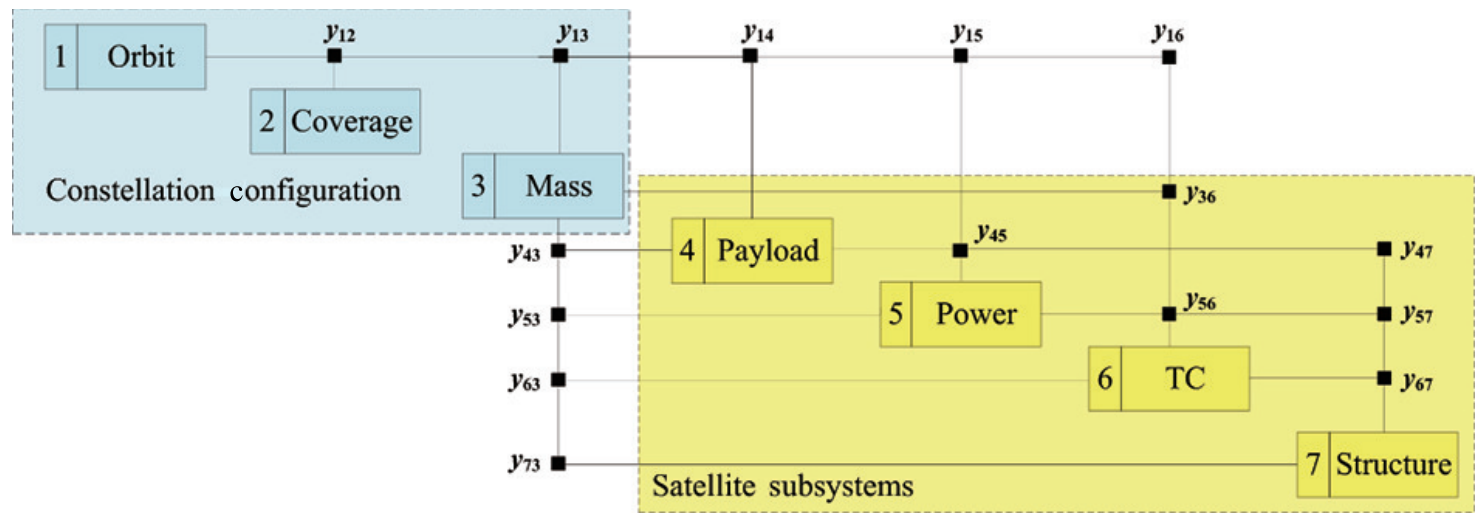

Fig. 18 Design structure matrix of satellite constellation MDO problem (reproduced with permission from Ref. [20], C) Springer-Verlag GmbH Germany, part of Springer Nature 2018).

optical parameters of the payload, power subsystem parameters, and thickness of structural plates. The discrete design variables $\left(\boldsymbol{X}_{\mathrm{d}}\right)$ include the number of orbit planes $(P)$ and the number of satellites in each orbit plane $(S)$. These design variables are optimized to minimize the total mass of the entire constellation system, subject to the constraints of coverage ratio, payload resolution, signal-to-noise ratio of payload, depth of discharge, power surplus, maximum temperature, and natural frequencies.

In this example, the sequential radial basis function method using a support vector machine for discretecontinuous mixed variables is used to solve the MDO problem. In the optimization method, a discrete-continuous variable sampling method is utilized to handle the discrete variables. The optimization results, summarized in Table 5 [20], are compared with those from the integer coding-based genetic algorithm. This indicates that the sequential radial basis function method considerably decreases the system mass by approximately $28.63 \%$. Moreover, the computational budget of this method com-

Table 5 System mass of optimized designs

\begin{tabular}{ccc}
\hline Parameters & $\begin{array}{c}\text { Integer } \\
\text { coding-based } \\
\text { genetic algorithm }\end{array}$ & $\begin{array}{c}\text { Sequential radial } \\
\text { basis function } \\
\text { method for } \\
\text { discrete- } \\
\text { continuous mixed } \\
\text { variables }\end{array}$ \\
\hline $\begin{array}{c}\text { Mass of single } \\
\text { satellite } \\
\text { Total mass of } \\
\text { satellite } \\
\text { constellation } \\
\text { system } \\
\text { Number of } \\
\text { simulations }\end{array}$ & $330.4 \mathrm{~kg}$ & $314.5 \mathrm{~kg}$ \\
\hline
\end{tabular}

pared with the integer coding-based genetic algorithm is reduced by more than $85 \%$. The optimized configurations of the constellations are shown in Fig. 19 [20].

In addition to the aforementioned metamodel-based aerospace system MDO examples, metamodeling techniques have also been widely used in the aerospace field. For instance, Xiong et al. proposed an intelligent optimization strategy based on statistical machine learning for spacecraft thermal design, where a neural network metamodel was used to approximate the spacecraftthermophysical model [179]. Smith et al. established a charged drag coefficient metamodel for spacecraft deorbit design with ionospheric drag from a low Earth orbit [180]. Wu et al. developed a double-layer radial basis function metamodel-based optimization method for the liquidgas interface determination of on-orbit refueling [181]. Peng and Wang proposed a dynamic metamodel-based optimization method for spacecraft formation reconfiguration on liberation point orbits to achieve fast path planning [182]. Feldhacker et al. investigated a samplingbased least-squares regression method to optimize the required velocity increments for rendezvous in spacecraft three-body dynamical systems [183]. Peng et al. proposed an adaptive metamodel-based optimization method called the sequential radial basis function for satellite truss structure optimization [184]. Gogu et al. proposed a response surface approximation method to aid in the design of integrated thermal protection systems for spacecraft reentry [185]. From the literature, MBDO methods are reported as effective and promising for solving complex aerospace system optimization problems. Simpson et al. applied Kriging models to an aerospike nozzle MDO problem for global approximation [186].

(10) $\underset{\text { UNIVERSITY PRESS }}{\operatorname{TSINGHA}}$ Springer 


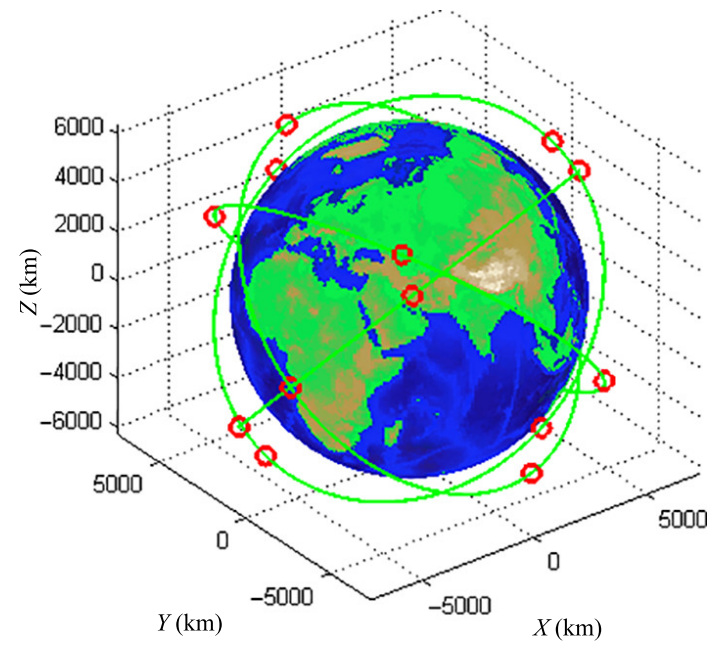

(a) Optimized design by integer coding-based genetic algorithm

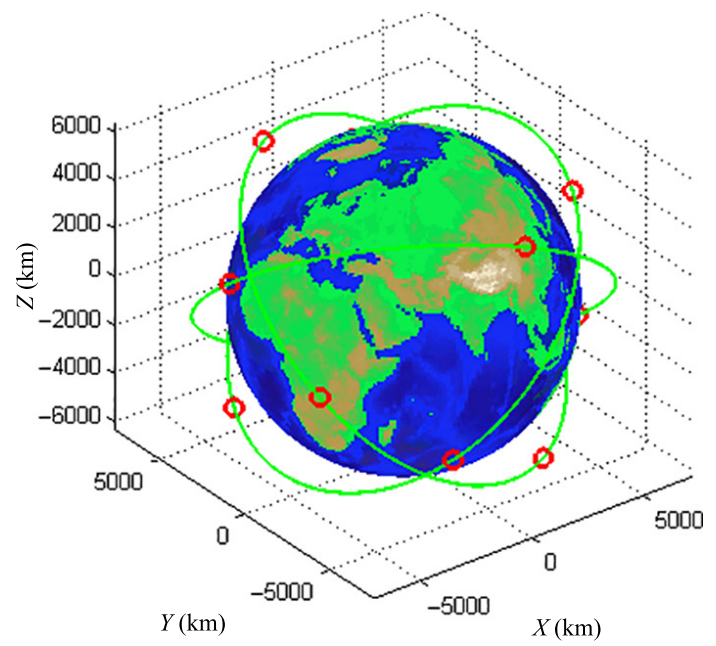

(b) Design by sequential radial basis function method

Fig. 19 Comparison of constellation configurations (reproduced with permission from Ref. [20], (C) Springer-Verlag GmbH Germany, part of Springer Nature 2018).

\section{Conclusions}

In this paper, an overview of the metamodel-based MDO and its application to aerospace systems is presented. The formulation of the MDO problem for aerospace systems and the overall procedure of the metamodel-based optimization process are introduced. Moreover, the technical architecture of metamodel-based MDO is summarized. Metamodeling techniques, including design of experiments, metamodels, and multi-model fusion methods, are surveyed with the aim of constructing a reducedorder numerical approximation model to inexpensively predict system responses during optimization. A number of state-of-the-art metamodel-based multidisciplinary op- timization strategies are discussed in detail. Generally, metamodel-based optimization strategies can be classified into two categories: infill criterion-based and space reduction-based optimization strategies. Recently, novel techniques, such as evolution computation and machine learning, have also been integrated with metamodels to further improve optimization performance. Finally, some penalty-based and penalty-free constraint-handling mechanisms are discussed to illustrate how to deal with expensive black-box constraints in the metamodel-based optimization process. Additionally, several real-world aerospace system MDO examples, which can aid researchers in realizing the applications of metamodel-based MDO in engineering practice, are presented. Note that although the contents of this paper are focused on the MDO for aerospace systems, the fundamental methodology and technology can also be applied to the design of other engineering systems, such as ships, aircraft, and automobiles, without significant modifications.

In future work, the optimization capacity of metamodel-based MDO must be further improved. For instance, due to the "curse of dimensionality", highdimensional expensive black-box problems remain complex for metamodel-based optimization. In addition, conventional metamodel-based MDOs generally consider multidisciplinary models as black-box functions. By exploiting prior knowledge (e.g., designer's experience, explicit model expression, and physical laws), the black-box MDO problem can be transformed into a gray-box or even a white-box optimization problem to further reduce cost and improve convergence. Moreover, the combination of metamodeling, MDO, and artificial intelligence has also attracted considerable interest. Thus, with the development of advanced modeling, analysis, and computation technologies, the research on metamodel-based MDO is anticipated to remain attractive.

\section{Acknowledgements}

This work was supported by the National Natural Science Foundation of China (Nos. 52005288 and 51675047) and the Aeronautical Science Foundation of China (No. 2019ZC072003).

\section{References}

[1] Sobieszczanski-Sobieski, J., Haftka, R. T. Multidisciplinary aerospace design optimization: Survey of recent 
developments. Structural Optimization, 1997, 14(1): $1-23$.

[2] Sobieszczanski-Sobieski, J. Multidisciplinary design optimization: Attempt at definition. In: Proceedings of the Industry University Workshop on Multidisciplinary Aircraft Design, 1993: 23-48.

[3] Martins, J. R. R. A., Lambe, A. B. Multidisciplinary design optimization: A survey of architectures. AIAA Journal, 2013, 51(9): 2049-2075.

[4] Grossman, B., Gurdal, Z., Strauch, G. J., Eppard, W. M., Haftka, R. T. Integrated aerodynamic/structural design of a sailplane wing. Journal of Aircraft, 1988, 25(9): 855-860.

[5] Livne, E., Schmit, L. A., Friedmann, P. P. Towards integrated multidisciplinary synthesis of actively controlled fiber composite wings. Journal of Aircraft, 1990, 27(12): 979-992.

[6] Wang, S., He, L. The summarization multidisciplinary design optimization for flying vehicles. Journal of Astronautics, 2004, 25(6): 697-701. (in Chinese)

[7] Chai, X., Yu, X., Wang, Y. Multipoint optimization on fuel efficiency in conceptual design of wide-body aircraft. Chinese Journal of Aeronautics, 2018, 31(1): 99-106.

[8] Liu, Y. B., Chen, B. Y., Li, Y. H., Shen, H. D. Overview of control-centric integrated design for hypersonic vehicles. Astrodynamics, 2018, 2(4): 307-324.

[9] Giannella, V., Lombardi, R., Pisani, M. M., Federico, L., Barbarino, M., Citarella, R. A novel optimization framework to replicate the vibro-acoustics response of an aircraft fuselage. Applied Sciences, 2020, 10(7): 2473.

[10] Wang, W., Wu, Z., Wang, D., Yang, J., Wang, P., Zhang, W. Hypersonic vehicle aerodynamic optimization using field metamodel-enhanced sequential approximate optimization. International Journal of Aerospace Engineering, 2021, 2021: 8889593.

[11] Wang, H., Shan, S., Wang, G. G., Li, G. Integrating least square support vector regression and mode pursuing sampling optimization for crashworthiness design. Journal of Mechanical Design, 2011, 133(4): 041002.

[12] Henric, P., Maria, R., Gerald, M., Hamid, H. Development of synthesis level design model in automobile application suitable for MDO using $\mathrm{CO}$ approach. $S A E$ International Journal of Materials and Manufacturing, 2015, 8(2): 344-356.

[13] Xue, Z., Adarsh, E., Fang, J. Multidisciplinary design optimization of vehicle weight reduction. SAE International Journal of Materials \& Manufacturing, 2016, 9(2): 393-399.
[14] Lai, G., Lei, J., Liu, J., Cao, S., Qin, H., Zeng, F., Zhou, R. Numerical and experimental study on comprehensive optimization for the KPIs of ship propulsion shafting design based on MDO. Ocean Engineering, 2021, 222: 108624 .

[15] Liu, Q., Feng, B., Liu, Z. Ship MDO modelling based on design structure matrix. Journal of South China University of Technology (Natural Science Edition), 2015, 43(04): 69-77. (in Chinese)

[16] Lei, G., Zhu, J., Guo, Y. Multidisciplinary Design Optimization Methods for Electrical Machines and Drive Systems. Springer-Verlag Berlin Heidelberg, 2016.

[17] Lei, G., Zhu, J., Guo, Y., Liu, C., Ma, B. A review of design optimization methods for electrical machines. Energies, 2017, 10(12): 1962.

[18] Wang, Y., Teng, H. Knowledge fusion design method: Satellite module layout. Chinese Journal of Aeronautics, 2009, 22(1): 32-42.

[19] Shi, R., Liu, L., Long, T., Liu, J., Yuan, B. Surrogate assisted multidisciplinary design optimization for an all-electric GEO satellite. Acta Astronautica, 2017, 138: $301-317$.

[20] Shi, R., Liu, L., Long, T., Wu, Y., Wang, G. G. Multidisciplinary modeling and surrogate assisted optimization for satellite constellation systems. Structural and Multidisciplinary Optimization, 2018, 58(5): 21732188.

[21] Shi, R., Liu, L., Long, T., Wu, Y., Tang, Y. Filter-based sequential radial basis function method for spacecraft multidisciplinary design optimization. AIAA Journal, 2018, 57(3): 1019-1031.

[22] Feng, X. Multidisciplinary design optimisation methods and their application in the design of navigation constellations. Ph.D. Dissertation. Changsha, China: University of Defence Science and Technology, 2008.

[23] Guo, S., Zhou, W. M., Zhang, J., Sun, F. Y., Yu, D. T. Integrated constellation design and deployment method for a regional augmented navigation satellite system using piggyback launches. Astrodynamics, 2021, 5(1): 49-60.

[24] Brown, N., Olds, J. Evaluation of multidisciplinary optimazation (MDO) techniques applied to a reusable launch vehicle. In: Proceedings of the 43rd AIAA Aerospace Sciences Meeting and Exhibit, 2005: AIAA 2005-707.

[25] Castellini, F., Lavagna, M. R., Riccardi, A., Büskens, C. Quantitative assessment of multidisciplinary design models for expendable launch vehicles. Journal of Spacecraft and Rockets, 2013, 51(1): 343-359.

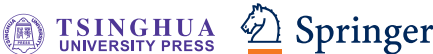


[26] Zhang, P., Yang, J., Luo, Z., Li, X. Collaborative optimization design of suspended rocket based on response surface. Journal of Solid Rocket Technology, 2017, 40(6): 793-798. (in Chinese)

[27] Liu, Z., Zhang, B. An overview of multidisciplinary optimization method in launch vehicle design. Astronautical Systems Engineering Technology, 2017, 1(2): 1-6.

[28] Wang, G. G., Shan, S. Review of metamodeling techniques in support of engineering design optimization. Journal of Mechanical Design, 2007, 129(4): 370-380.

[29] Forrester, A. I. J., Keane, A. J. Recent advances in surrogate-based optimization. Progress in Aerospace Sciences, 2009, 45(1-3): 50-79.

[30] Han, Z., Xu, C., Qiao, J., Liu, F., Chi, J., Meng, G., Zhang, K., Song, W. Recent progress of efficient global aerodynamic shape optimization using surrogate-based approach. Acta Aeronautica et Astronautica Sinica, 2020, 41(5): 31-47. (in Chinese)

[31] De Weck, O., Agte, J., Sobieszczanski-Sobieski, J., Arendsen, P., Morris, A., Spieck, M. State-of-the-art and future trends in multidisciplinary design optimization. In: Proceedings of the 48th AIAA/ASME/ASCE/ AHS/ASC Structures, Structural Dynamics, and Materials Conference, 2007: AIAA 2007-1905.

[32] Simpson, T. W., Toropov, V., Balabanov, V., Viana, F. A. C. Design and analysis of computer experiments in multidisciplinary design optimization: A review of how far we have come - or not. In: Proceedings of the 12th AIAA/ISSMO Multidisciplinary Analysis and Optimization Conference, 2008: AIAA 2008-5802.

[33] Queipo, N. V., Haftka, R. T., Shyy, W., Goel, T., Vaidyanathan, R., Kevin Tucker, P. Surrogate-based analysis and optimization. Progress in Aerospace Sciences, 2005, 41(1): 1-28.

[34] Viana, F. A. C., Simpson, T. W., Balabanov, V., Toropov, V. Special section on multidisciplinary design optimization: Metamodeling in multidisciplinary design optimization: How far have we really come? AIAA Journal, 2014, 52(4): 670-690.

[35] Simpson, T. W., Booker, A. J., Ghosh, D., Giunta, A. A., Koch, P. N., Yang, R. J. Approximation methods in multidisciplinary analysis and optimization: A panel discussion. Structural and Multidisciplinary Optimization, 2004, 27(5): 302-313.

[36] Long, T., Liu, J., Wang, G. G., Liu, L., Shi, R., Guo, X. Discuss on approximate optimization strategies using design of computer experiments and metamodels for flight vehicle design. Journal of Mechanical Engineering, 2016, 52(14): 79-105. (in Chinese)
[37] Johnson, M. E., Moore, L. M., Ylvisaker, D. Minimax and maximin distance designs. Journal of Statistical Planning and Inference, 1990, 26(2): 131-148.

[38] Koehler, J. R., Owen, A. B. 9 computer experiments. Handbook of Statistics, 1996, 13: 261-308.

[39] Bates, S., Sienz, J., Toropov, V. Formulation of the optimal Latin hypercube design of experiments using a permutation genetic algorithm. In: Proceedings of the 45th AIAA/ASME/ASCE/AHS/ASC Structures, Structural Dynamics \& Materials Conference, 2004: AIAA 2004-2011.

[40] Tanabe, R., Fukunaga, A. S. Improving the search performance of shade using linear population size reduction. In: Proceedings of the 2014 IEEE Congress on Evolutionary Computation New York, 2014: 16581665.

[41] Jin, R., Chen, W., Sudjianto, A. An efficient algorithm for constructing optimal design of computer experiments. Journal of Statistical Planning and Inference, 2005, 134(1): 268-287.

[42] Ye, K. Q., Li, W., Sudjianto, A. Algorithmic construction of optimal symmetric Latin hypercube designs. Journal of Statistical Planning and Inference, 2000, 90(1): 145-159.

[43] Zhu, H., Liu, L., Long, T., Peng, L. A novel algorithm of maximin Latin hypercube design using successive local enumeration. Engineering Optimization, 2012, 44(5): 551-564.

[44] Viana, F. A. C., Venter, G., Balabanov, V. An algorithm for fast optimal Latin hypercube design of experiments. International Journal for Numerical Methods in Engineering, 2010, 82(2): 135-156.

[45] Gunst, R. F. Response surface methodology: process and product optimization using designed experiments. Technometrics, 1996, 38(3): 284-286.

[46] Buhmann, M. D. Radial basis functions: Theory and implementations. Cambridge: Cambridge university press, 2003.

[47] Viana, F. A. C., Haftka, R. T., Steffen, V. Multiple surrogates: How cross-validation errors can help us to obtain the best predictor. Structural and Multidisciplinary Optimization, 2009, 39(4): 439-457.

[48] Song, X., Lv, L., Li, J., Sun, W., Zhang, J. An advanced and robust ensemble surrogate model: Extended adaptive hybrid functions. Journal of Mechanical Design, 2018, 140(4): 041402.

[49] Acar, E. Simultaneous optimization of shape parameters and weight factors in ensemble of radial basis functions. Structural and Multidisciplinary Optimization, 2014, 49(6): 969-978. 
[50] Clarke, S. M., Griebsch, J. H., Simpson, T. W. Analysis of support vector regression for approximation of complex engineering analyses. Journal of Mechanical Design, Transactions of the ASME, 2005, 127(6): 1077-1087.

[51] Awad, M., Khanna, R. Support vector regression. In: Efficient Learning Machines: Theories, Concepts, and Applications for Engineers and System Designers. Berkeley, CA: Apress. 2015: 67-80.

[52] Haykin, S. Neural networks: A comprehensive foundation. New York: Macmillan College Publishing Company, 1998.

[53] Damianou, A., Lawrence, N. D. Deep Gaussian processes. In: Proceedings of the 16th International Conference on Artificial Intelligence and Statistics, 2013.

[54] Salimbeni, H., Deisenroth, M. P. Doubly stochastic variational inference for deep Gaussian processes. In: Proceedings of the 31st International Conference on Neural Information Processing Systems, 2017.

[55] Rajaram, D., Puranik, T. G., Ashwin Renganathan, S., Sung, W., Fischer, O. P., Mavris, D. N., Ramamurthy, A. Empirical assessment of deep Gaussian process surrogate models for engineering problems. Journal of Aircraft, 2020, 58(1): 182-196.

[56] Forrester, A. I. J., Sóbester, A., Keane, A. J. Multifidelity optimization via surrogate modelling. Proceedings of the Royal Society A: Mathematical, Physical and Engineering Science, 2007, 463: 3251-3269.

[57] Park, C., Haftka, R. T., Kim, N. H. Remarks on multifidelity surrogates. Structural and Multidisciplinary Optimization, 2017, 55(3): 1029-1050.

[58] Le Gratiet, L. Multi-fidelity Gaussian process regression for computer experiments, Université Paris-Diderot Paris VII, 2013.

[59] Zhou, Q., Yang, Y., Song, X., Han, Z., Cheng, Y., Hu, J., Shu, L., Jiang, P. Survey of multi-fidelity surrogate models and their applications in the design and optimization of engineering equipment. Journal of Mechanical Engineering, 2020, 56(24): 219-245. (in Chinese)

[60] Liu, H., Ong, Y. S., Cai, J., Wang, Y. Cope with diverse data structures in multi-fidelity modeling: A Gaussian process method. Engineering Applications of Artificial Intelligence, 2018, 67: 211-225.

[61] Fischer, C. C., Grandhi, R. V. Utilizing an adjustment factor to scale between multiple fidelities within a design process: A stepping stone to dialable fidelity design. In: Proceedings of the 16th AIAA Non-Deterministic Approaches Conference, 2014: AIAA 2014-1011.

[62] Zheng, J., Shao, X. Y., Gao, L., Jiang, P., Li, Z. L. A hybrid variable-fidelity global approximation modelling method combining tuned radial basis function base and kriging correction. Journal of Engineering Design, 2013, 24(8): 604-622.

[63] Kennedy, M., O'Hagan, A. Predicting the output from a complex computer code when fast approximations are available. Biometrika, 2000, 87(1): 1-13.

[64] Qian, P. Z. G., Wu, C. F. J. Bayesian hierarchical modeling for integrating low-accuracy and high-accuracy experiments. Technometrics, 2008, 50(2): 192-204.

[65] Le Gratiet, L. Recursive co-kriging model for Design of Computer experiments with multiple levels of fidelity with an application to hydrodynamic. 2012: arXiv: 1210.0686[math.ST]. Available at https://arxiv.org/ abs/1210.0686.

[66] Han, Z. H., Görtz, S. Hierarchical kriging model for variable-fidelity surrogate modeling. AIAA Journal, 2012, 50(9): 1885-1896.

[67] Zhang, Y., Han, Z. H., Zhang, K. S. Variable-fidelity expected improvement method for efficient global optimization of expensive functions. Structural and Multidisciplinary Optimization, 2018, 58(4): 1431-1451.

[68] Han, Z. H., Görtz, S. Alternative cokriging method for variable-fidelity surrogate modeling. AIAA Journal, 2012, 50(5): 1205-1210.

[69] Bertram, A., Zimmermann, R. Theoretical investigations of the new Cokriging method for variablefidelity surrogate modeling. Advances in Computational Mathematics, 2018, 44(6): 1693-1716.

[70] Toal, D. J. J. Some considerations regarding the use of multi-fidelity Kriging in the construction of surrogate models. Structural and Multidisciplinary Optimization, 2015, 51(6): 1223-1245.

[71] Jones, D. R., Schonlau, M., Welch, W. J. Efficient global optimization of expensive black-box functions. Journal of Global Optimization, 1998, 13(4): 455-492.

[72] Shi, R., Liu, L., Long, T., Wu, Y., Tang, Y. Filterbased adaptive Kriging method for black-box optimization problems with expensive objective and constraints. Computer Methods in Applied Mechanics and Engineering, 2019, 347: 782-805.

[73] Long, T., Wei, Z., Shi, R. H., Wu, Y. F. Parallel adaptive kriging method with constraint aggregation for expensive black-box optimization problems. AIAA Journal, 2021: 1-15.

[74] Amine Bouhlel, M., Bartoli, N., Regis, R. G., Otsmane, A., Morlier, J. Efficient global optimization for highdimensional constrained problems by using the Kriging models combined with the partial least squares method. Engineering Optimization, 2018, 50(12): 2038-2053.

[75] Xu, S. G., Chen, H. Q., Zhang, J. L. A study of NashEGO algorithm for aerodynamic shape design optimizations. Structural and Multidisciplinary Optimization, 2019, 59(4): 1241-1254. 
[76] Viana, F. A. C., Haftka, R. T., Watson, L. T. Efficient global optimization algorithm assisted by multiple surrogate techniques. Journal of Global Optimization, 2013, 56(2): 669-689.

[77] He, Y., Sun, J., Song, P., Wang, X. Variable-fidelity expected improvement based efficient global optimization of expensive problems in presence of simulation failures and its parallelization. Aerospace Science and Technology, 2021, 111: 106572.

[78] Zhan, D. W., Xing, H. L. Expected improvement for expensive optimization: A review. Journal of Global Optimization, 2020, 78(3): 507-544.

[79] Wang, L., Shan, S., Wang, G. G. Mode-pursuing sampling method for global optimization on expensive black-box functions. Engineering Optimization, 2004, 36(4): 419-438.

[80] Branin, F. H. J., Hoo, S. K. A method for finding multiple extrema of a function of $\mathrm{n}$ variables. Numerical Methods, 1972: 231-237.

[81] Wu, Y. F., Long, T., Shi, R. H., Wang, G. G. Modepursuing sampling method using discriminative coordinate perturbation for high-dimensional expensive blackbox optimization. Journal of Mechanical Design, 2021, 143(4): 041703.

[82] Kazemi, M., Wang, G. G., Rahnamayan, S., Gupta, $\mathrm{K}$. Constraint importance mode pursuing sampling for continuous global optimization. In: Proceedings of the ASME 2010 International Design Engineering Technical Conferences and Computers and Information in Engineering Conference, 2011: 325-334.

[83] Sharif, B., Wang, G. G., Elmekkawy, T. Y. Mode pursuing sampling method for discrete variable optimization on expensive black-box functions. Journal of Mechanical Design, 2008, 130(2): 021402.

[84] Wang, G. G., Shan, S. Q. An efficient Pareto set identification approach for multi-objective optimization on black-box functions. In: Proceedings of the ASME 2004 International Design Engineering Technical Conferences and Computers and Information in Engineering Conference, 2008: 279-291.

[85] Cheng, G. H., Younis, A., Haji Hajikolaei, K., Wang, G. G. Trust region based mode pursuing sampling method for global optimization of high dimensional design problems. Journal of Mechanical Design, 2015, 137(2): 021407.

[86] Cheng, G. H., Gjernes, T., Wang, G. G. An adaptive aggregation-based approach for expensively constrained black-box optimization problems. Journal of Mechanical Design, 2018, 140(9): 091402.
[87] Booker, A. J., Dennis, J. E., Frank, P. D., Serafini, D. B., Torczon, V., Trosset, M. W. A rigorous framework for optimization of expensive functions by surrogates. Structural Optimization, 1999, 17(1): 1-13.

[88] Jones, D. R. A taxonomy of global optimization methods based on response surfaces. Journal of Global $\mathrm{Op}$ timization, 2001, 21(4): 345-383.

[89] Watson, A. G., Barnes, R. J. Infill sampling criteria to locate extremes. Mathematical Geology, 1995, 27(5): 589-608.

[90] Keane, A. J., Forrester, A. I. J., Sóbester, A. Engineering Design via Surrogate Modelling: A Practical Guide. John Wiley \& Sons, Ltd., 2008.

[91] Martin, J., Simpson, T. W. Use of adaptive metamodeling for design optimization. In: Proceedings of the 9th AIAA/ISSMO Symposium on Multidisciplinary Analysis and Optimization, 2002: 2002-5631.

[92] van Beers, W. C. M., Kleijnen, J. P. C. Kriging for interpolation in random simulation. Journal of the $\mathrm{Op}$ erational Research Society, 2003, 54(3): 255-262.

[93] Cox, D. D., John, S. A statistical method for global optimization. In: Proceedings of the 1992 IEEE International Conference on Systems, Man, and Cybernetics, 1992: 1241-1246.

[94] Long, T., Liu, L., Peng, L. Global optimization method with enhanced adaptive response surface method for computation-intensive design problems. Advanced Science Letters, 2012, 5(2): 881-887.

[95] Jiang, P., Cheng, J., Zhou, Q., Shu, L. S., Hu, J. X. Variable-fidelity lower confidence bounding approach for engineering optimization problems with expensive simulations. AIAA Journal, 2019, 57(12): 5416-5430.

[96] Zhu, H. G., Liu, L., Long, T., Zhao, J. F. Global optimization method using SLE and adaptive RBF based on fuzzy clustering. Chinese Journal of Mechanical Engineering, 2012, 25(4): 768-775.

[97] Tian, K., Li, Z. C., Huang, L., Du, K. F., Jiang, L. L., Wang, B. Enhanced variable-fidelity surrogate-based optimization framework by Gaussian process regression and fuzzy clustering. Computer Methods in Applied Mechanics and Engineering, 2020, 366: 113045.

[98] Zhou, Y. C., Lu, Z. Z. An enhanced Kriging surrogate modeling technique for high-dimensional problems. $\mathrm{Me}$ chanical Systems and Signal Processing, 2020, 140: 106687.

[99] Han, Z. Kriging surrogate model and its application to design optimization: A review of recent progress. Acta Aeronautica et Astronautica Sinica, 2016, 37(11): 3197-3225. (in Chinese) 
[100] Gao, Y., Wang, X. A sequential optimization method with multi-point sampling criterion based on Kriging surrogate model. Engineering Mechanics, 2012, 29(4): 90-95. (in Chinese)

[101] Song, C., Yang, X. D., Song, W. P. Multi-infill strategy for kriging models used in variable fidelity optimization. Chinese Journal of Aeronautics, 2018, 31(3): 448-456.

[102] Alexandrov, N. M., Dennis, J. E., Lewis, R. M., Torczon, V. A trust-region framework for managing the use of approximation models in optimization. Structural Optimization, 1998, 15(1): 16-23.

[103] Pérez, V. M., Renaud, J. E., Watson, L. T. Adaptive experimental design for construction of response surface approximations. AIAA Journal, 2002, 40(12): 24952503.

[104] Gano, S. E., Renaud, J. E., Martin, J. D., Simpson, T. W. Update strategies for kriging models used in variable fidelity optimization. Structural and Multidisciplinary Optimization, 2006, 32(4): 287-298.

[105] Cheng, G., Wang, G. F. Trust region based MPS method for global optimization of high dimensional design problems. In: Proceedings of the 53rd AIAA/ ASME/ASCE/AHS/ASC Structures, Structural Dynamics and Materials Conference, 2012: AIAA 20121290.

[106] Shi, R. H., Liu, L., Long, T., Liu, J. Sequential radial basis function using support vector machine for expensive design optimization. AIAA Journal, 2016, 55(1): 214-227.

[107] Suthaharan, S. Support vector machine. In: Machine Learning Models and Algorithms for Big Data Classification. Boston, MA: Springer US, 2016: 207-235.

[108] Rish, I. An empirical study of the naive Bayes classifier. In: Proceedings of the IJCAI 2001 Workshop on Empirical Methods in Artificial Intelligence, 2001.

[109] Wang, G. G., Dong, Z. M., Aitchison, P. Adaptive response surface method - a global optimization scheme for approximation-based design problems. Engineering Optimization, 2001, 33(6): 707-733.

[110] Long, T., Wu, D., Guo, X. S., Wang, G. G., Liu, L. Efficient adaptive response surface method using intelligent space exploration strategy. Structural and Multidisciplinary Optimization, 2015, 51(6): 13351362 .

[111] Long, T., Liu, L., Peng, L., Li, Y. Aero-structure coupled optimization of high aspect ratio wing using enhanced adaptive response surface method. In: Proceedings of the 12th AIAA Aviation Technology, Integration, and Operations Conference and 14th AIAA/ISSMO Multidisciplinary Analysis and Optimization Conference, 2012: AIAA 2012-5456.
[112] Wu, D., Long, T., Li, Y. L., Jiang, M. L., Huang, B. Aero-structure-stealth coupled optimization for high aspect ratio wing using adaptive metamodeling method. 15th AIAA/ISSMO Multidisciplinary Analysis and Optimization Conference, 2014.

[113] Dong, H. C., Sun, S. Q., Song, B. W., Wang, P. Multisurrogate-based global optimization using a score-based infill criterion. Structural and Multidisciplinary Optimization, 2019, 59(2): 485-506.

[114] Qiu, H. B., Xu, Y. J., Gao, L., Li, X. K., Chi, L. Multi-stage design space reduction and metamodeling optimization method based on self-organizing maps and fuzzy clustering. Expert Systems With Applications, 2016, 46: 180-195.

[115] Liu, H. T., Xu, S. L., Wang, X. F. Sequential sampling designs based on space reduction. Engineering Optimization, 2015, 47(7): 867-884.

[116] Jin, Y. C. Surrogate-assisted evolutionary computation: Recent advances and future challenges. Swarm and Evolutionary Computation, 2011, 1(2): 61-70.

[117] Jin, Y. C., Wang, H. D., Chugh, T., Guo, D., Miettinen, K. Data-driven evolutionary optimization: An overview and case studies. IEEE Transactions on Evolutionary Computation, 2019, 23(3): 442-458.

[118] Li, F., Shen, W. M., Cai, X. W., Gao, L., Wang, G. G. A fast surrogate-assisted particle swarm optimization algorithm for computationally expensive problems. Applied Soft Computing, 2020, 92: 106303.

[119] Yang, Z., Qiu, H. B., Gao, L., Cai, X. W., Jiang, C., Chen, L. M. Surrogate-assisted classificationcollaboration differential evolution for expensive constrained optimization problems. Information Sciences, 2020, 508: 50-63.

[120] Wang, X. J., Wang, G. G., Song, B. W., Wang, P., Wang, Y. A novel evolutionary sampling assisted optimization method for high-dimensional expensive problems. IEEE Transactions on Evolutionary Computation, 2019, 23(5): 815-827.

[121] Tian, J., Tan, Y., Zeng, J. C., Sun, C. L., Jin, Y. C. Multiobjective infill criterion driven Gaussian process-assisted particle swarm optimization of highdimensional expensive problems. IEEE Transactions on Evolutionary Computation, 2019, 23(3): 459-472.

[122] Wang, H. D., Jin, Y. C., Doherty, J. Committee-based active learning for surrogate-assisted particle swarm optimization of expensive problems. IEEE Transactions on Cybernetics, 2017, 47(9): 2664-2677.

[123] Glaz, B., Friedmann, P. P., Liu, L., Cajigas, J. G., Bain, J., Sankar, L. N. Reduced-order dynamic stall modeling with swept flow effects using a surrogate-based recurrence framework. AIAA Journal, 2013, 51(4): 910-921. 
[124] Sun, C. L., Zeng, J. C., Pan, J., Xue, S. D., Jin, Y. C. A new fitness estimation strategy for particle swarm optimization. Information Sciences, 2013, 221: 355370.

[125] Vincenzi, L., Gambarelli, P. A proper infill sampling strategy for improving the speed performance of a Surrogate-Assisted Evolutionary Algorithm. Computers Es Structures, 2017, 178: 58-70.

[126] Miranda-Varela, M. E., Mezura-Montes, E. Constrainthandling techniques in surrogate-assisted evolutionary optimization. An empirical study. Applied Soft Computing, 2018, 73: 215-229.

[127] Wang, Y., Yin, D. Q., Yang, S. X., Sun, G. Y. Global and local surrogate-assisted differential evolution for expensive constrained optimization problems with inequality constraints. IEEE Transactions on Cybernetics, 2019, 49(5): 1642-1656.

[128] Zhao, X. G., Zhang, K., Chen, G. D., Xue, X. M., Yao, C. J., Wang, J., Yang, Y. F., Zhao, H., Yao, J. Surrogate-assisted differential evolution for production optimization with nonlinear state constraints. Journal of Petroleum Science and Engineering, 2020, 194: 107441.

[129] Zhang, K., Zhao, X. G., Chen, G. D., Zhao, M. J., Wang, J., Yao, C. J., Sun, H., Yao, J., Wang, W., Zhang, G. D. A double-model differential evolution for constrained waterflooding production optimization. Journal of Petroleum Science and Engineering, 2021, 207: 109059.

[130] Yu, M. Y., Li, X., Liang, J. A dynamic surrogateassisted evolutionary algorithm framework for expensive structural optimization. Structural and Multidisciplinary Optimization, 2020, 61(2): 711-729.

[131] Lim, D. K., Woo, D. K., Yeo, H. K., Jung, S. Y., Ro, J. S., Jung, H. K. A novel surrogate-assisted multiobjective optimization algorithm for an electromagnetic machine design. IEEE Transactions on Magnetics, 2015, 51(3): 1-4.

[132] Datta, R., Regis, R. G. A surrogate-assisted evolution strategy for constrained multi-objective optimization. Expert Systems With Applications, 2016, 57: 270-284.

[133] Díaz-Manríquez, A., Toscano, G., Barron-Zambrano, J. H., Tello-Leal, E. A review of surrogate assisted multiobjective evolutionary algorithms. Computational Intelligence and Neuroscience, 2016, 2016: 1-14.

[134] Venturelli, G., Benini, E., Łaniewski-Wołtk, E. A Kriging-assisted multiobjective evolutionary algorithm. Applied Soft Computing, 2017, 58: 155-175.

[135] Wang, C. Z., Ding, J. L., Cheng, R., Liu, C. X., Chai, T. Y. Data-driven surrogate-assisted multi-objective optimization of complex beneficiation operational process. IFAC-PapersOnLine, 2017, 50(1): 14982-14987.
[136] Chugh, T., Jin, Y. C., Miettinen, K., Hakanen, J., Sindhya, K. A surrogate-assisted reference vector guided evolutionary algorithm for computationally expensive many-objective optimization. IEEE Transactions on Evolutionary Computation, 2018, 22(1): 129-142.

[137] Wan, K. Z., He, C., Camacho, A., Shang, K., Cheng, R., Ishibuchi, H. A hybrid surrogate-assisted evolutionary algorithm for computationally expensive manyobjective optimization. In: Proceedings of the 2019 IEEE Congress on Evolutionary Computation, 2019: 2018-2025.

[138] Liu, B., Koziel, S., Zhang, Q. F. A multi-fidelity surrogate-model-assisted evolutionary algorithm for computationally expensive optimization problems. Journal of Computational Science, 2016, 12: 28-37.

[139] Yi, J., Shen, Y. C., Shoemaker, C. A. A multi-fidelity RBF surrogate-based optimization framework for computationally expensive multi-modal problems with application to capacity planning of manufacturing systems. Structural and Multidisciplinary Optimization, 2020, 62(4): 1787-1807.

[140] Xie, T. L., Jiang, P., Zhou, Q., Shu, L. S., Zhang, Y. H., Meng, X. Z., Wei, H. Advanced multi-objective robust optimization under interval uncertainty using kriging model and support vector machine. Journal of Computing and Information Science in Engineering, 2018, 18(4): 041012.

[141] Malak, R. J. Jr, Paredis, C. J. J. Using support vector machines to formalize the valid input domain of predictive models in systems design problems. Journal of Mechanical Design, 2010, 132(10): 101001.

[142] Liu, X., Wu, Y. Z., Wang, B. X., Ding, J. W., Jie, H. X. An adaptive local range sampling method for reliabilitybased design optimization using support vector machine and Kriging model. Structural and Multidisciplinary Optimization, 2017, 55(6): 2285-2304.

[143] Tang, Y. F., Long, T., Shi, R. H., Wu, Y. F., Wang, G. G. Sequential radial basis function-based optimization method using virtual sample generation. Journal of Mechanical Design, 2020, 142(11): 111701.

[144] Basudhar, A., Dribusch, C., Lacaze, S., Missoum, S. Constrained efficient global optimization with support vector machines. Structural and Multidisciplinary $O p$ timization, 2012, 46(2): 201-221.

[145] Lim, D., Ong, Y. S., Gupta, A., Goh, C. K., Dutta, P. S. Towards a new Praxis in optinformatics targeting knowledge re-use in evolutionary computation: Simultaneous problem learning and optimization. Evolutionary Intelligence, 2016, 9(4): 203-220.

[146] Park, J., Kim, K. Y. Instance variant nearest neighbor using particle swarm optimization for function approximation. Applied Soft Computing, 2016, 40: 331-341. 
[147] Isaacs, A., Ray, T., Smith, W. An evolutionary algorithm with spatially distributed surrogates for multiobjective optimization. In: Progress in Artificial Life. ACAL 200\%. Lecture Notes in Computer Science, Vol. 4828. Randall, M., Abbass, H. A., Wiles, J., Eds. Springer, Berlin, Heidelberg, 2007: 257-268

[148] Castillo, E., Mínguez, R., Castillo, C. Sensitivity analysis in optimization and reliability problems. Reliability Engineering \& System Safety, 2008, 93(12): 1788-1800.

[149] Chen, G., Zhang, K., Xue, X., Zhang, L., Yao, J., Sun, H., Fan, L., Yang, Y. Surrogate-assisted evolutionary algorithm with dimensionality reduction method for water flooding production optimization. Journal of Petroleum Science and Engineering, 2020, 185: 106633.

[150] Liu, B., Zhang, Q., Gielen, G. G. A Gaussian process surrogate model assisted evolutionary algorithm for medium scale expensive optimization problems. IEEE Transactions on Evolutionary Computation, 2013, 18(2): 180-192.

[151] Gupta, A., Ong, Y., Feng, L. Insights on transfer optimization: Because experience is the best teacher. IEEE Transactions on Emerging Topics in Computational Intelligence, 2018, 2(1): 51-64.

[152] Gupta, A., Ong, Y., Feng, L., Tan, K. C. Multiobjective multifactorial optimization in evolutionary multitasking. IEEE Transactions on Cybernetics, 2017, 47(7): 1652-1665.

[153] Ma, X., Chen, Q., Yu, Y., Sun, Y., Ma, L., Zhu, Z. A two-level transfer learning algorithm for evolutionary multitasking. Frontiers in Neuroscience, 2020, 13: 1408.

[154] Cheng, M.-Y., Gupta, A., Ong, Y.-S., Ni, Z.-W. Coevolutionary multitasking for concurrent global optimization: With case studies in complex engineering design. Engineering Applications of Artificial Intelligence, 2017, 64: 13-24.

[155] Min, A. T. W., Ong, Y., Gupta, A., Goh, C. Multiproblem surrogates: Transfer evolutionary multiobjective optimization of computationally expensive problems. IEEE Transactions on Evolutionary Computation, 2019, 23(1): 15-28.

[156] Raissi, M., Perdikaris, P., Karniadakis, G. E. Physicsinformed neural networks: A deep learning framework for solving forward and inverse problems involving nonlinear partial differential equations. Journal of Computational Physics, 2019, 378: 686-707.

[157] Karniadakis, G. E., Kevrekidis, I. G., Lu, L., Perdikaris, P., Wang, S., Yang, L. Physics-informed machine learning. Nature Reviews Physics, 2021, 3(6): 422-440.
[158] Lu, L., Jin, P., Pang, G., Zhang, Z., Karniadakis, G. E. Learning nonlinear operators via DeepONet based on the universal approximation theorem of operators. Nature Machine Intelligence, 2021, 3(3): 218-229.

[159] Kashefi, A., Rempe, D., Guibas, L. J. A point-cloud deep learning framework for prediction of fluid flow fields on irregular geometries. Physics of Fluids, 2021, 33(2): 027104.

[160] Kondor, R., Son, H. T., Pan, H., Anderson, B., Trivedi, S. Covariant compositional networks for learning graphs. 2018: arXiv:180102144. Available at https: //arxiv.org/abs/2009.11193.

[161] Darbon, J., Meng, T. On some neural network architectures that can represent viscosity solutions of certain high dimensional Hamilton-Jacobi partial differential equations. Journal of Computational Physics, 2021, 425: 109907.

[162] Chen, W., Chiu, K., Fuge, M. D. Airfoil design parameterization and optimization using bézier generative adversarial networks. AIAA Journal, 2020, 58(11): 47234735.

[163] Pfrommer, S., Halm, M., Posa, M. Contactnets: Learning of discontinuous contact dynamics with smooth, implicit representations. 2020: arXiv:200911193. Available at https://arxiv.org/abs/2009.11193.

[164] Wang, G. G., Simpson, T. Fuzzy clustering based hierarchical metamodeling for design space reduction and optimization. Engineering Optimization, 2004, 36(3): 313-335.

[165] Fletcher, R., Leyffer, S. Nonlinear programming without a penalty function. Mathematical Programming, 2002, 91(2): 239-269.

[166] Fletcher, R., Leyffer, S., Toint, P. L. On the global convergence of a filter-SQP algorithm. SIAM Journal on Optimization, 2002, 13(1): 44-59.

[167] Audet, C., Dennis, J. E. A pattern search filter method for nonlinear programming without derivatives. SIAM Journal on Optimization, 2004, 14(4): 980-1010.

[168] Gonzaga, C. C., Karas, E., Vanti, M. A globally convergent filter method for nonlinear programming. SIAM Journal on Optimization, 2004, 14(3): 646-669.

[169] Regis, R. G. Constrained optimization by radial basis function interpolation for high-dimensional expensive black-box problems with infeasible initial points. Engineering Optimization, 2014, 46(2): 218-243.

[170] Rashid, K., Ambani, S., Cetinkaya, E. An adaptive multiquadric radial basis function method for expensive black-box mixed-integer nonlinear constrained optimization. Engineering Optimization, 2013, 45(2): 185206. 
[171] Qian, J. C., Yi, J. X., Cheng, Y. S., Liu, J., Zhou, Q. A sequential constraints updating approach for Kriging surrogate model-assisted engineering optimization design problem. Engineering With Computers, 2020, 36(3): 993-1009.

[172] Sasena, M. J., Papalambros, P., Goovaerts, P. Exploration of metamodeling sampling criteria for constrained global optimization. Engineering Optimization, 2002, 34(3): 263-278.

[173] Kreisselmeier, G., Steinhauser, R. Systematic control design by optimizing a vector performance index. IFAC Proceedings Volumes, 1979, 12(7): 113-117.

[174] Poon, N. M. K., Martins, J. R. R. A. An adaptive approach to constraint aggregation using adjoint sensitivity analysis. Structural and Multidisciplinary Optimization, 2007, 34(1): 61-73.

[175] Gao, H., Zhu, J., Zhang, W., Zhou, Y. An improved adaptive constraint aggregation for integrated layout and topology optimization. Computer Methods in Applied Mechanics and Engineering, 2015, 289: 387-408.

[176] Zhang, K. S., Han, Z. H., Gao, Z. J., Wang, Y. Constraint aggregation for large number of constraints in wing surrogate-based optimization. Structural and Multidisciplinary Optimization, 2019, 59(2): 421-438.

[177] Kennedy, G. J. Strategies for adaptive optimization with aggregation constraints using interior-point methods. Computers \& Structures, 2015, 153: 217-229.

[178] Shi, R., Liu, L., Long, T., Liu, J. An efficient ensemble of radial basis functions method based on quadratic programming. Engineering Optimization, 2016, 48(7): $1202-1225$.

[179] Xiong, Y., Guo, L., Tian, D., Zhang, Y., Liu, C. Intelligent optimization strategy based on statistical machine learning for spacecraft thermal design. IEEE Access, 2020, 8: 204268-204282.

[180] Smith, B. G. A., Capon, C. J., Brown, M., Boyce, R. R. Ionospheric drag for accelerated deorbit from upper low earth orbit. Acta Astronautica, 2020, 176: 520-530.

[181] Wu, Z., Huang, Y., Chen. X., Zhang, X., Yao, W. Surrogate modeling for liquid-gas interface determination under microgravity. Acta Astronautica, 2018, 152: 71-77.

[182] Peng, H., Wang. W. Adaptive surrogate model-based fast path planning for spacecraft formation reconfiguration on libration point orbits. Aerospace Science and Technology, 2016, 54: 151-163.

[183] Feldhacker, J. D., Jones, B. A., Doostan, A., Hampton, J. Reduced cost mission design using surrogate models. Advances in Space Research, 2016, 57(2): 588-603.
[184] Peng, L., Liu, L., Long, T., Yang, W. An efficient truss structure optimization framework based on CAD/CAE integration and sequential radial basis function metamodel. Structural and Multidisciplinary Optimization, 2014, 50(2): 329-346.

[185] Gogu, C., Haftka, R. T., Bapanapalli, S. K., Sankar, B. V. Dimensionality reduction approach for response surface approximations: Application to thermal design. AIAA Journal, 2009, 47(7): 1700-1708.

[186] Simpson, T. W., Mauery, T. M., Korte, J. J., Mistree, F. Kriging models for global approximation in simulation-based multidisciplinary design optimization. AIAA Journal, 2001, 39(12): 2233-2241.

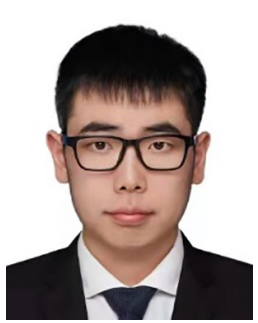

Renhe Shi received his B.S. degree in flight vehicle design engineering and $\mathrm{Ph} . \mathrm{D}$. degree in aeronautical and astronautical science and technology from Beijing Institute of Technology, China, in 2013 and 2019, respectively. $\mathrm{He}$ is a postdoctoral researcher in the School of Aerospace Engineering, Tsinghua University, from 2019 to 2020. Currently, he is an associate professor in the School of Aerospace Engineering, Beijing Institute of Technology. His research interests include metamodel-based design optimization, multidisciplinary design optimization, and aerospace system engineering. E-mail: shirenhe@bit.edu.cn.

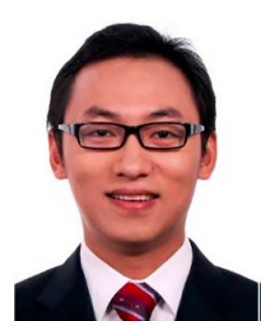

Teng Long received his B.S. degree in flight vehicle propulsion engineering and Ph.D. degree in aeronautical and astronautical science and technology from Beijing Institute of Technology, China, in 2004 and 2009, respectively. He is a professor and executive dean at the School of Aerospace Engineering, Beijing Institute of Technology. His research interests include multidisciplinary design optimization theory and its applications to flight vehicle conceptual design and multi-aircraft collaborative mission planning and decision-making. Professor Long is a member of the American Institute of Aeronautics and Astronautics (AIAA) and the American Society of Mechanical Engineers (ASME). E-mail: tenglong@bit.edu.cn. 


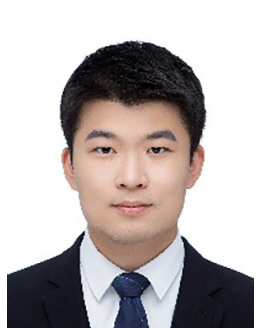

Nianhui Ye received his B.S. degree in flight vehicle design and engineering from Beijing Institute of Technology, China, in 2019. He is currently pursuing his Ph.D. degree at the School of Aerospace and Engineering, Beijing Institute of Technology. His research interests include surrogateassisted evolutionary computation and multidisciplinary modeling of cross-media flight vehicles. E-mail: ynh1996sh@163.com.

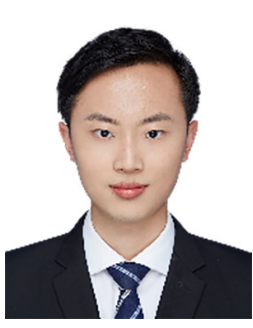

Yufei Wu received his B.S. degree in flight vehicle design engineering from Beijing Institute of Technology, China, in 2017. He is currently pursuing his Ph.D. degree in aeronautical and astronautical science and technology at Beijing Institute of Technology. His research interests include metamodel-based design optimization theory and its applications to cross-domain morphing flight vehicle design. E-mail: wuyufei@bit.edu.cn.

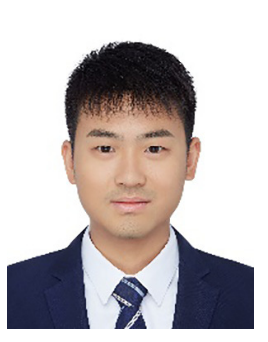

Zhao Wei received his B.S. degree in mechanical engineering and automation from Zhengzhou University, China, in 2016. He is currently pursuing his Ph.D. degree in aeronautical and astronautical science and technology at Beijing Institute of Technology. His research interests include flight vehicle multidisciplinary design optimization and mission planning. E-mail: weizhao0123@163.com.

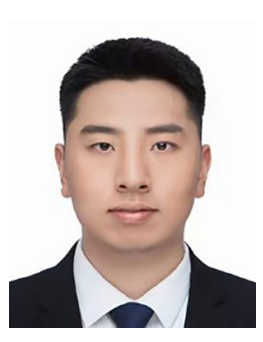

Zhenyu Liu received his B.S. degree in flight vehicle design and engineering from Beijing Institute of Technology, China, in 2020. He is currently pursuing his M.S. degree at the School of Aerospace and Engineering, Beijing Institute of Technology, China. His research interests include surrogate-assisted multi-objective optimization methods and flight vehicle multidisciplinary design optimization. E-mail: liuzykm@163.com.

Open Access This article is licensed under a Creative Commons Attribution 4.0 International License, which permits use, sharing, adaptation, distribution and reproduction in any medium or format, as long as you give appropriate credit to the original author(s) and the source, provide a link to the Creative Commons licence, and indicate if changes were made.

The images or other third party material in this article are included in the article's Creative Commons licence, unless indicated otherwise in a credit line to the material. If material is not included in the article's Creative Commons licence and your intended use is not permitted by statutory regulation or exceeds the permitted use, you will need to obtain permission directly from the copyright holder.

To view a copy of this licence, visit http://creativecommons.org/licenses/by/4.0/. 